\title{
Analyzing the contribution of individual resonance poles of the $S$-matrix to the two-channel scattering
}

\author{
S. A. Rakityansky* and N. Elander** \\ ${ }^{*}$ Dept. of Physics, University of Pretoria, Lynnwood Road, Pretoria 0002, South Africa \\ ** Div. of Molecular Physics, Dept. of Physics, Stockholm University, Stockholm, SE-106 91, Sweden
}

August 14, 2018

\begin{abstract}
A two-channel problem is considered within a method based on first order differential equations that are equivalent to the corresponding Schrödinger equation but are more convenient for dealing with resonant phenomena. Using these equations, it is possible to directly calculate the Jost matrix for practically any complex value of the energy. The spectral points (bound and resonant states) can therefore be located in a rigorous way, namely, as zeros of the Jost matrix determinant. When calculating the Jost matrix, the differential equations are solved and thus, at the same time, the wave function is obtained with the correct asymptotic behavior that is embedded in the solution analytically. The method offers very accurate way of calculating not only total widths of resonances but their partial widths as well. For each pole of the $S$-matrix, its residue can be calculated rather accurately, which makes it possible to obtain the Mittag-Leffler type expansion of the $S$-matrix as a sum of the singular terms (representing the resonances) and the background term (contour integral). As an example, the two-channel model by Noro and Taylor is considered. It is demonstrated how the contributions of individual resonance poles to the scattering cross section can be analyzed using the Mittag-Leffler expansion and the Argand plot technique. This example shows that even poles situated far away from the physical real axis may give significant contributions to the cross section.
\end{abstract}

PACS number(s): 03.65.Nk, 03.65.-w, 31.15.-p, 34.50.-s 
KEY WORDS: two-channel scattering, quantum resonances, Jost-matrix, complex rotation, Argand plot, partial widths

\section{Published in: Int.J.Quant.Chem.,106(2006)1105-1129}

\section{Introduction}

Mankind has always been fascinated by striking phenomena such as thunderstorms, earthquakes, and comets. As a part of mankind, scientists also are more attracted to drastically rather than smoothly varying quantities. Quantum physicists considering collisions of the molecular, atomic or sub-atomic particles, when find irregularities in their energy dependencies, try to assign certain quantum numbers to the outstanding features. These and other phenomena can often be regarded as resonances. Friedmann and Truhlar point out that "Probably the most striking phenomenon in the whole range of scattering experiments is the resonance" [1]. However, not all irregularities in the scattering data are true resonances [2].

Early studies of the energy dependence of nuclear scattering that led Bohr [3] to the idea of the compound nucleus (for more elaborate discussions see, for example, Ref. [4]), inspired similar ideas to appear in chemical physics. The notion of collision complex, used in atomic and molecular physics as well as chemistry, was developed in theoretical studies of the early 1970s [5, 6] (see also Ref. [7]).

However, it took about 20 years for indirect experimental evidence of the existence of such complexes to appear [8, 9]. The first direct observation of an isolated resonance in chemical reactions only appeared around 2000 [7, 10. Still it is not absolutely clear that the results of Ref. [10] concerning the existence of the FHD collision complex in the $F+H D \rightarrow F H D \rightarrow F H+D$ reaction, give an undoubted evidence. For example, in Ref.[1] the doubts are expressed in terms of the definition of a resonance, which is rigorously defined as a quantum state that at large distances is a purely outgoing wave. Mathematically, this implies that the matrix element of the operator that transforms the incoming wave into the outgoing wave (the $S$-operator), has a pole at the resonance (complex valued) energy [12, 13].

A theoretical analysis of intermediate states, in the collisions like $F+H D$, will eventually need more than one electronic potential energy surface, a feature which is not easily achieved[14]. It is thus, from a theoretical point of view, reasonable to begin with atom ion collisions, which can be represented by a set of coupled potential energy curves.

Looking back in the literature, we find that a few resonances were found in a theoretical study of the $N^{3+}+H \rightarrow N^{2+}+H^{+}$reaction by McCarroll and Valrion[15]. Bárány et 
al. [16] and Rittby et al. [17] extended this work and could assign complex eigenenergies and their rovibrational quantum numbers to not only the resonances found in [15]. These resonances were, as in the work by Elander and coworkers [18, 19, 20, 21], forming a sting that started with a narrow resonance and propagated out in the complex energy plane. The group lead by Shimakura, studied several charge-transfer reactions with the initial channel $N^{5+}+H$ (see Refs. [22, 23, 24, 25]) while Bacchys-Montabonel and Ceyzeriat [26] did calculations for the ion-atom collision $\mathrm{Si}^{4+}+\mathrm{He}$. Both groups predicted resonances in these reactions. Experimentalists at Oak Ridge made an effort to find evidence for these type of resonances [27, 28] but the obtainable energy range and resolution did not permit their detection. The conclusion was that due to extremely high Coulomb barrier the relative energy $(\sim \mathrm{meV})$ at which these resonances appear, could never be achieved in collision experiments.

The interest to the resonant charge-transfers and other similar processes did not wane. The new idea is to observe the collisions between particles moving in the same beam, so that their relative kinetic energy is very low. Electrostatic storage rings for this purpose are currently being built. For example, the Desiree project in Stockholm [29] is planned to have the possibility of merging a stored multiply charged ion beam with a beam of neutral atoms. Similar experiments are also planned at other facilities.

In the theoretical studies, the most ubiquitous formula associated with resonance phenomena, is the one bearing the names of Breit and Wigner. It was invented for phenomenological description of the peaks in the neutron scattering cross section [30] and is valid in the neighborhood of an isolated resonance. From textbooks, we learn that if a resonance is embedded in a direct scattering continuum, the cross section profile does not have a Lorentian shape as predicted by the Breit-Wigner formula, but should rather be described by the more general Butler-Fano parameters [31].

The original ideas of Breit and Wigner [30] and Fano [31] may make us believe that resonances only give a signature to a cross section as peaks or similar irregularities. However, model potential studies of the Schrödinger spectrum [32, 33] found that the $S$-matrix poles associated with resonances, form long (most likely infinite) strings in the complex energy plane. The influence of these poles on the cross section could be more complicated than simple generating of the peaks or bumps. The resonances may interfere with each other and thus cause an intricate energy dependence of the cross section.

The most controversial example of such intricate interference of resonance states is the problem of barrier states in chemical physics. According to Friedmann and Truhlar, "metastable states associated with barriers, are associated with poles of the S-matrix just as definitely as are trapped states associated with standing waves in a well" and these metastable states are the bottle necks for chemical reactions (see Refs. [1] and [34]). In relation to barrier scattering reactions like $H+H D \rightarrow D+H_{2}$, these ideas are dis- 
cussed by Althrope et al. [35], where they report "an intriguing forward-scattering peak" observed at a collision energy $\sim 1.64 \mathrm{eV}$ in a state-to-state differential cross section. From the point of view of Friedmann and Truhlar [1], this observation could be a manifestation of quantum barrier states suggested by them. This is opposed by Manolopoulos [36] who claims that isolated (i.e. true) resonances and barrier type resonances are "mathematically different and the physical implications of this mathematical difference are too significant to regard the two situations as a manifestation of the same phenomenon" [35]. Other similar results on "quantized bottleneck state(s)" are reported in Ref. [37]. It should also be mentioned that the quantized bottleneck ideas do have implications on the understanding of the so called transition states discussed in chemical physics ( See Ref. [38] for a review).

The controversies, like the barrier states, can only be resolved if we reach a clear understanding of how much a given isolated or barrier type resonance influences the cross section. The importance of this is emphasized by the authors of Refs. [1, 34, 35, 36, 37] and many others. A number of methods were developed for the purpose of analyzing the roles of individual resonances in the scattering cross section. A comparison between the Mittag-Leffler expansion approach (see, for example, Ref.[18]), the Siegert pseudo-state method [39], and the complex Kohn variational method was recently published in Ref. [40]. Based on the Titchmarsh-Weyl theory, the authors of Ref. [41] developed a method for decomposing the scattering information into the resonance and background contributions. One of us (N.E.) has earlier analyzed the Mittag-Leffler method to understand its computational properties [19, 20, 21]. In Ref. [21], we also investigated the influence of a string of resonance poles yielding somewhat surprising results.

One drawback of the Mittag-Leffler expansion method is that it may be hard to apply it to the systems involving more than two particles in at least one of the channels. In this respect the complex variational Kohn method, as described by Nuttal and Cohen [42, Rescigno and McCurdy[43], and recently in the review by Moiseyev [44], has an advantage. It can be formulated in terms of matrix elements of the interaction potential and the complex eigenvalues of the corresponding analytically continued Hamiltonian [44]. In this way the Kohn method is applicable whenever a basis set representation of the problem is available. It can therefore be extended to the 3-D problems such as, for example, the collisions complex FHD etc. [8, 9, 10]. However, being based on the basis expansion, the complex variational Kohn method has its disadvantages as well. These are related to the problem of convergence and completeness of the expansion. It is thus of importance to compare and analyze the three above mentioned methods in parallel to understand and utilize their respective powers in analyzing and predicting experimental results.

As a prerequisite for the analysis of the cross sections of chemical reactions, one needs 
a reliable method for solving the multi-channel Schrödinger problem. As we already mentioned, in theoretical analysis of atomic complexes, like $F+H D$, it is very common to represent the atom-ion interaction by a set of coupled potential energy curves.

In this paper, we adopt a mathematically rigorous definition of a resonance, namely, as a point on the unphysical sheet of complex energy surface at which the Jost-matrix determinant is zero. At such points, the $S$-matrix has poles since this determinant is in the denominators of all its matrix elements. The so-called redundant (non-resonance) poles that the $S$-matrix may also have (see, for example, Ref.[45]), are thus avoided since they are associated with singularities of the numerators of its matrix elements. This mathematically-inclined definition is consistent with physics in that at thus defined resonance points the wave function has the Gamow-Siegert asymptotics (pure outgoing waves), but it does not say anything about observable irregularities of the cross section. Therefore the term "resonance" has here a more general meaning not limited to long-lived (narrow) states.

In the paper, we describe a mathematically rigorous and numerically accurate and stable method for solving the two-channel (generally, $N$-channel) quantum mechanical problem. For the last ten years various aspects of this method were developed in Refs. [46, 47, 48, 49, 50, 51, 52, 53, 54, 55, 56, 57, 58. Here we suggest an approach to analyzing the contribution of resonance $S$-matrix poles to the scattering picture. Our approach is based on a Mittag-Leffler type expansion of the $S$-matrix, for which we calculate the residues of the $S$-matrix at the resonance poles and its Cauchy-type contour integral. The roles played by individual resonances are deduced using the Argand plot technique. Practical application of the method is demonstrated by the example of the Noro and Taylor two-channel model [59].

\section{Two-channel Schrödinger equation}

Let us consider a quantum mechanical two-body problem which, after separation of the motion of its center of mass, is reduced to an effective problem of one body whose dynamics is governed by the Hamiltonian

$$
H=H_{0}+U+h
$$

where the terms on the right hand side describe its free motion $\left(H_{0}\right)$, the interaction forces $(U)$, and the internal dynamics in the body $(h)$, respectively. Since our main objective is to study how the resonance poles of the $S$-matrix manifest themselves in experimentally observable cross sections, we avoid unnecessary complications associated with spins of the particles and with long-range Coulomb forces. Furthermore, for the sake of clarity, we 
consider here a two-channel system, although all the equations can be easily generalized for an arbitrary number of channels (see Ref. [52]) as well as for charged particles with non-zero spins (see Refs. [48, 50]).

Therefore we assume that the colliding particles are neutral and spinless, and the Hamiltonian $h$ acts in the space spanned by only two of its eigenstates,

$$
h|n\rangle=E_{n}|n\rangle, \quad n=1,2,
$$

i. e.

$$
h=E_{1}|1\rangle\left\langle 1\left|+E_{2}\right| 2\right\rangle\langle 2| .
$$

In its turn, this implies that the operators $H_{0}$ and $U$ are $2 \times 2$ matrices in the subspace associated with the internal degrees of freedom. Therefore the total Hamiltonian (1) taken in the coordinate representation and sandwiched between $\langle n|$ and $\left|n^{\prime}\right\rangle$, has the following matrix representation

$$
H=\left(\begin{array}{cc}
-\frac{\hbar^{2}}{2 \mu_{1}} \Delta_{\vec{r}}+U_{11}(\vec{r})+E_{1} & U_{12}(\vec{r}) \\
U_{21}(\vec{r}) & -\frac{\hbar^{2}}{2 \mu_{2}} \Delta_{\vec{r}}+U_{22}(\vec{r})+E_{2}
\end{array}\right)
$$

where $\vec{r}$ is the relative coordinate and the subscripts label the channels. A solution of the corresponding Schrödinger equation

$$
H \psi=E \psi
$$

is a column matrix

$$
\psi=\left(\begin{array}{c}
\psi_{1}(E, \vec{r}) \\
\psi_{2}(E, \vec{r})
\end{array}\right)
$$

composed of the $\vec{r}$-dependent coefficients in the expansion of the state vector over the channel (internal) states, i.e.

$$
\langle\vec{r} \mid \Psi\rangle=\psi_{1}(E, \vec{r})|1\rangle+\psi_{2}(E, \vec{r})|2\rangle
$$

Continuing to keep unnecessary complications away, we assume that the interaction potentials are spherically symmetric which means that the orbital angular momentum $\ell$ associated with $\vec{r}$, is conserving and hence is the same for the in and out channels. Furthermore, for the matrix elements of the potential, we assume that

$$
\int_{0}^{\infty}\left|U_{n n^{\prime}}(r)\right| r d r<\infty,
$$


i.e. that their possible singularity at $r=0$ is less than $1 / r^{2}$ and they vanish at infinity faster than $1 / r^{2}$. Expanding the channel wave function in partial waves,

$$
\psi_{n}(E, \vec{r})=\frac{u_{n}(E, r)}{r} Y_{\ell m}(\hat{\vec{r}})
$$

we obtain a set of coupled radial Schrödinger equations

$$
\mathcal{H} u=V u
$$

where $u$ is a column matrix consisting of $u_{1}(E, r)$ and $u_{2}(E, r)$, matrix elements of $V$ are

$$
V_{n n^{\prime}}(r)=\frac{2 \mu_{n}}{\hbar^{2}} U_{n n^{\prime}}(r)
$$

and

$$
\mathcal{H}=\left(\begin{array}{cc}
\frac{d^{2}}{d r^{2}}+k_{1}^{2}-\frac{\ell(\ell+1)}{r^{2}} & 0 \\
0 & \frac{d^{2}}{d r^{2}}+k_{2}^{2}-\frac{\ell(\ell+1)}{r^{2}}
\end{array}\right)
$$

with the channel momenta $k_{n}$ defined as

$$
k_{n}^{2}=\frac{2 \mu_{n}}{\hbar^{2}}\left(E-E_{n}\right) .
$$

As is well known (from the general theory of ordinary differential equations), a coupled set of $N$ equations of the type (9) has $2 N$ linearly independent column solutions, and only half of them are regular at $r=0$. Since in our case $N=2$, we have two independent regular column-solutions that can be combined in a square $2 \times 2$ matrix

$$
\phi(E, r)=\left(\begin{array}{ll}
\phi_{11}(E, r) & \phi_{12}(E, r) \\
\phi_{21}(E, r) & \phi_{22}(E, r)
\end{array}\right),
$$

where the first subscript labels the channels and the second is the solution number. For the independent regular solutions, we use the symbol $\phi$ to distinguish them from the physical solutions $u$. By definition, they obey the same equation, namely,

$$
\mathcal{H} \phi=V \phi \text {. }
$$

Since all physical solutions $u(E, r)$ must be regular at $r=0$, anyone of them is a linear combination of the regular columns, i.e.

$$
\left(\begin{array}{c}
u_{1}(E, r) \\
u_{2}(E, r)
\end{array}\right)=\left(\begin{array}{c}
\phi_{11}(E, r) \\
\phi_{21}(E, r)
\end{array}\right) c_{1}+\left(\begin{array}{c}
\phi_{12}(E, r) \\
\phi_{22}(E, r)
\end{array}\right) c_{2}
$$


or in a more compact form

$$
\left(\begin{array}{l}
u_{1} \\
u_{2}
\end{array}\right)=\left(\begin{array}{ll}
\phi_{11} & \phi_{12} \\
\phi_{21} & \phi_{22}
\end{array}\right)\left(\begin{array}{l}
c_{1} \\
c_{2}
\end{array}\right) .
$$

The combination coefficients $c_{1}$ and $c_{2}$ should be chosen in such a way that guarantees a given (physical) behavior of the solution when $r \rightarrow \infty$.

\section{Transformation to first-order equations}

If the right hand side of Eq. (14) were zero, the equations of the set would be uncoupled (independent) and could be solved analytically. Indeed, the Riccati-Hankel functions $h_{\ell}^{( \pm)}\left(k_{n} r\right)$ are solutions of the equation

$$
\left[\frac{d^{2}}{d r^{2}}+k_{n}^{2}-\frac{\ell(\ell+1)}{r^{2}}\right] h_{\ell}^{( \pm)}\left(k_{n} r\right)=0 .
$$

Therefore the matrices

$$
W_{\ell}^{(\mathrm{in})}(E, r)=\left(\begin{array}{cc}
h_{\ell}^{(-)}\left(k_{1} r\right) & 0 \\
0 & h_{\ell}^{(-)}\left(k_{2} r\right)
\end{array}\right)
$$

and

$$
W_{\ell}^{(\text {out })}(E, r)=\left(\begin{array}{cc}
h_{\ell}^{(+)}\left(k_{1} r\right) & 0 \\
0 & h_{\ell}^{(+)}\left(k_{2} r\right)
\end{array}\right)
$$

both solve Eq. (14) when its right hand side is zero, i.e.

$$
\mathcal{H} W_{\ell}^{(\text {in/out })}(E, r)=0
$$

These matrices represent the incoming and outgoing spherical waves which form the asymptotics of the solution at large distances when the potential vanishes. In order to guarantee correct asymptotic behavior of the solutions (13) of Eq. (14), let us look for them in the following form

$$
\phi(E, r)=W_{\ell}^{(\text {in })}(E, r) \mathcal{F}^{(\text {in })}(E, r)+W_{\ell}^{(\text {out })}(E, r) \mathcal{F}^{(\text {out })}(E, r),
$$

where $\mathcal{F}^{\text {(in/out) }}(E, r)$ are new unknown matrix functions. In the theory of ordinary differential equations, this way of finding solution is known as the variation parameters method. 
Since we replaced one unknown matrix $\phi$ with two unknown matrices $\mathcal{F}^{\text {(in/out) }}$, they cannot be independent. We therefore can impose one arbitrary condition that relates them to each other. As such condition, we choose the following equation

$$
W_{\ell}^{(\mathrm{in})}(E, r) \frac{d}{d r} \mathcal{F}^{(\mathrm{in})}(E, r)+W_{\ell}^{\text {(out) }}(E, r) \frac{d}{d r} \mathcal{F}^{\text {(out) }}(E, r)=0
$$

which is standard in the variation parameters method and is called the Lagrange condition. It implies that, when calculating first derivative of $\phi(E, r)$, we only need to differentiate the spherical waves,

$$
\frac{d}{d r} \phi(E, r)=W_{\ell}^{\prime(\text { in })}(E, r) \mathcal{F}^{(\text {in })}(E, r)+W_{\ell}^{\prime(\text { out })}(E, r) \mathcal{F}^{(\text {out })}(E, r)
$$

Let us substitute the ansatz (20) into Eq. (14) and thus obtain the corresponding equations that determine $\mathcal{F}^{\text {(in) }}$ and $\mathcal{F}^{\text {(out) }}$. The second derivative of $\phi(E, r)$ is

$$
\frac{d^{2}}{d r^{2}} \phi=W_{\ell}^{\prime \prime(\text { in) }} \mathcal{F}^{(\text {in) }}+W_{\ell}^{\prime \prime(\text { out })} \mathcal{F}^{\text {(out) }}+W_{\ell}^{\prime(\text { in) }} \mathcal{F}^{\prime(\text { in })}+W_{\ell}^{\prime(\text { out })} \mathcal{F}^{\prime(\text { out })} .
$$

This means that

$$
\mathcal{H} \phi=\left(\mathcal{H} W_{\ell}^{(\text {in })}\right) \mathcal{F}^{(\text {in })}+\left(\mathcal{H} W_{\ell}^{(\text {in })}\right) \mathcal{F}^{(\text {in })}+W_{\ell}^{\prime(\text { in })} \mathcal{F}^{\prime(\text { in })}+W_{\ell}^{\prime(\text { out })} \mathcal{F}^{\prime(\text { out })}
$$

where the first two terms disappear in accordance with Eq. (19). Therefore Eq. (14) takes the form

$$
W_{\ell}^{\prime(\text { in })} \mathcal{F}^{(\text {in })}+W_{\ell}^{\prime(\text { out })} \mathcal{F}^{\prime(\text { out })}=V\left[W_{\ell}^{(\text {in })} \mathcal{F}^{(\text {in })}+W_{\ell}^{(\text {out })} \mathcal{F}^{(\text {out })}\right] .
$$

From the Lagrange condition (21), it follows that

$$
\mathcal{F}^{\prime(\text { out })}=-\left[W_{\ell}^{(\text {out })}\right]^{-1} W_{\ell}^{(\text {in })} \mathcal{F}^{\prime(\text { in })}
$$

It should be noted that since matrices $W_{\ell}^{(\text {in/out })}$ and their derivatives all are diagonal, they commute with each other. Substituting Eq. (24) into Eq. (23) and using this commutative property, we obtain

$$
\mathcal{F}^{\prime(\text { in })}=\left[W_{\ell}^{(\text {out })} W_{\ell}^{\prime(\text { in })}-W_{\ell}^{\prime(\text { out })} W_{\ell}^{(\text {in })}\right]^{-1} W_{\ell}^{(\text {out })} V\left[W_{\ell}^{(\text {in })} \mathcal{F}^{(\text {in })}+W_{\ell}^{(\text {out })} \mathcal{F}^{(\text {out })}\right] .
$$

Similarly, from the Lagrange condition (21), it follows that

$$
\mathcal{F}^{\prime(\text { in })}=-\left[W_{\ell}^{(\text {in })}\right]^{-1} W_{\ell}^{(\text {out })} \mathcal{F}^{\prime(\text { out })}
$$


and therefore Eq. (23) gives

$$
\mathcal{F}^{\prime(\text { out })}=\left[W_{\ell}^{(\text {in })} W_{\ell}^{\prime(\text { out })}-W_{\ell}^{\prime(\text { in })} W_{\ell}^{(\text {out })}\right]^{-1} W_{\ell}^{(\text {in })} V\left[W_{\ell}^{(\text {in })} \mathcal{F}^{(\text {in })}+W_{\ell}^{(\text {out })} \mathcal{F}^{(\text {out })}\right] .
$$

The first factors in Eqs. (25) and (27) differ only by the sign and actually are equal to the inverted Wronskian of the incoming and outgoing spherical waves, which can be found in explicit form. Indeed, knowing the Wronskian of the Riccati-Hankel functions,

$$
h_{\ell}^{(-)}(k r) \frac{d}{d r} h_{\ell}^{(+)}(k r)-\frac{d}{d r} h_{\ell}^{(-)}(k r) h_{\ell}^{(+)}(k r)=2 i k
$$

we obtain

$$
W_{\ell}^{(\text {in })} W_{\ell}^{\prime(\text { out })}-W_{\ell}^{\prime(\text { in })} W_{\ell}^{(\text {out })}=2 i K
$$

where

$$
K=\left(\begin{array}{cc}
k_{1} & 0 \\
0 & k_{2}
\end{array}\right) .
$$

Therefore the Schrödinger equation (14) is equivalent to the following set of coupled equations of the first order

$$
\left\{\begin{array}{l}
\frac{d}{d r} \mathcal{F}^{\text {(in) }}=-\frac{1}{2 i} K^{-1} W_{\ell}^{(\text {out })} V\left[W_{\ell}^{(\text {in })} \mathcal{F}^{(\text {in })}+W_{\ell}^{(\text {out })} \mathcal{F}^{(\text {out })}\right] \\
\frac{d}{d r} \mathcal{F}^{\text {(out) }}=\frac{1}{2 i} K^{-1} W_{\ell}^{(\text {in })} V\left[W_{\ell}^{(\text {in })} \mathcal{F}^{(\text {in })}+W_{\ell}^{(\text {out })} \mathcal{F}^{(\text {out })}\right]
\end{array} .\right.
$$

Boundary conditions at $r=0$ for equations (31) follow from the requirement that the solution (20) must be regular. This can only be achieved if $\mathcal{F}^{\text {(in) }}(E, r)$ and $\mathcal{F}^{\text {(out) }}(E, r)$ become identical when $r \rightarrow 0$ because in such a case the singular parts of $h_{\ell}^{(-)}(k r)$ and $h_{\ell}^{(+)}(k r)$ cancel each other, i.e.

$$
h_{\ell}^{(-)}(k r)+h_{\ell}^{(+)}(k r)=2 j_{\ell}(k r) .
$$

Here the Riccati-Bessel function $j_{\ell}$ is regular, namely,

$$
j_{\ell}(k r) \underset{r \rightarrow 0}{\longrightarrow} \frac{\sqrt{\pi}}{2^{\ell+1} \Gamma(\ell+3 / 2)}(k r)^{\ell+1} .
$$

Matrices $\mathcal{F}^{(\text {in })}(E, r)$ and $\mathcal{F}^{(\text {out })}(E, r)$ must become diagonal near $r=0$ in order to guarantee linear independence of the columns of $\phi(E, r)$. Thus, the following boundary conditions

$$
\lim _{r \rightarrow 0} \mathcal{F}_{n n^{\prime}}^{\text {(in/out) }}(E, r)=\delta_{n n^{\prime}}
$$

are appropriate since we have the freedom to choose the normalization of the solution. 


\section{Jost matrices}

The differential equations (31) can be numerically solved from the origin to a sufficiently far point $r=R$ where the potential vanishes (causing the right-hand sides of the equations to disappear) and therefore $\mathcal{F}^{\text {(in/out) }}(E, r)$ become constant. These constants

$$
f^{(\text {in })}(E)=\lim _{r \rightarrow \infty} \mathcal{F}^{(\text {in })}(E, r)
$$

and

$$
f^{\text {(out) }}(E)=\lim _{r \rightarrow \infty} \mathcal{F}^{\text {(out) }}(E, r)
$$

are the Jost matrices that determine asymptotic behavior of the fundamental system of regular solutions

$$
\phi(E, r) \underset{r \rightarrow \infty}{\longrightarrow} W_{\ell}^{(\text {in })}(E, r) f^{(\text {in })}(E)+W_{\ell}^{(\text {out })}(E, r) f^{(\text {out })}(E) .
$$

It is worthwhile to mention that we use here the notation $f^{\text {(in/out) }}(E)$ which is different from the traditional notation such as $f^{( \pm)}( \pm k)$. There are two reasons for this. First of all, we do not fix the normalization of the regular solution $\phi(E, r)$. As a result, both $f^{(\text {in })}(E)$ and $f^{(\text {out })}(E)$ can have an arbitrary common factor. We are not concerned with this factor because no observable quantity depends on it. In contrast to a majority of other studies, we leave the normalization of the Jost matrices free, and therefore need a notation that is different from the traditional. The second reason is that the superscripts $(\mathrm{in})$ and (out) are unambiguous and thus we avoid possible confusion caused by the existence of notations with opposite signs for the same Jost matrices.

The regular solution $\phi(E, r)$ consists of two linearly independent columns which constitute the fundamental system of solutions. Any other solution is a linear combination of these independent columns. The physical wave function (15) is one of such combinations,

$$
u(E, r)=\phi(E, r) c
$$

where

$$
u=\left(\begin{array}{l}
u_{1} \\
u_{2}
\end{array}\right) \quad \text { and } \quad c=\left(\begin{array}{l}
c_{1} \\
c_{2}
\end{array}\right) .
$$

At large distances, we therefore have

$$
u(E, r) \underset{r \rightarrow \infty}{\longrightarrow} W_{\ell}^{(\mathrm{in})}(E, r) f^{(\mathrm{in})}(E)\left(\begin{array}{c}
c_{1} \\
c_{2}
\end{array}\right)+W_{\ell}^{(\mathrm{out})}(E, r) f^{(\mathrm{out})}(E)\left(\begin{array}{l}
c_{1} \\
c_{2}
\end{array}\right) .
$$




\subsection{Spectral points}

There are certain discrete points in the complex $E$-plane (bound, resonant, and virtual states), at which the physical wave function has only outgoing waves in its asymptotic behavior. At these so called spectral points the combination coefficients $c_{1}$ and $c_{2}$ are such that

$$
\left(\begin{array}{cc}
f_{11}^{(\mathrm{in})}(E) & f_{12}^{(\mathrm{in})}(E) \\
f_{21}^{(\mathrm{in})}(E) & f_{22}^{(\mathrm{in})}(E)
\end{array}\right)\left(\begin{array}{c}
c_{1} \\
c_{2}
\end{array}\right)=0 .
$$

This homogeneous equation has a non-trivial solution if and only if

$$
\operatorname{det} f^{(\mathrm{in})}(E)=0
$$

As is seen, the distribution of the spectral points in the complex E-plane (determined by this equation) does not, as was mentioned earlier, depend on the normalization of the Jost matrix.

\subsection{Scattering}

For a scattering state, the wave function has both incoming and outgoing waves at large $r$. If $A_{n}$ and $B_{n}$ are the amplitudes of the incoming and outgoing (scattered) waves in the $n$-th channel, then the scattering boundary condition (at $r \rightarrow \infty$ ) reads

$$
\begin{aligned}
u(E, r) \underset{r \rightarrow \infty}{\longrightarrow}\left(\begin{array}{c}
h_{\ell}^{(-)}\left(k_{1} r\right) A_{1}+h_{\ell}^{(+)}\left(k_{1} r\right) B_{1} \\
h_{\ell}^{(-)}\left(k_{2} r\right) A_{2}+h_{\ell}^{(+)}\left(k_{2} r\right) B_{2}
\end{array}\right) \\
=W_{\ell}^{(\text {in) }}(E, r)\left(\begin{array}{c}
A_{1} \\
A_{2}
\end{array}\right)+W_{\ell}^{(\text {out })}(E, r)\left(\begin{array}{c}
B_{1} \\
B_{2}
\end{array}\right) .
\end{aligned}
$$

Comparing Eqs. (42) and (39), we see that

$$
\left(\begin{array}{c}
A_{1} \\
A_{2}
\end{array}\right)=f^{(\mathrm{in})}(E)\left(\begin{array}{l}
c_{1} \\
c_{2}
\end{array}\right)
$$

and

$$
\left(\begin{array}{c}
B_{1} \\
B_{2}
\end{array}\right)=f^{(\text {out })}(E)\left(\begin{array}{c}
c_{1} \\
c_{2}
\end{array}\right) .
$$


This implies that the amplitudes of the incident and scattered waves are related by a $2 \times 2$-matrix , $S(E)$ as

$$
\left(\begin{array}{l}
B_{1} \\
B_{2}
\end{array}\right)=S(E)\left(\begin{array}{c}
A_{1} \\
A_{2}
\end{array}\right)
$$

This $S$-matrix

$$
S(E)=f^{(\text {out })}(E)\left[f^{(\text {in })}(E)\right]^{-1}
$$

does not depend on the choice of $A_{n}$. We can therefore clarify the physical meaning of its matrix elements by considering special cases with simple choices of $A_{1}$ and $A_{2}$. If the incident wave is purely channel 1 , i.e. $A_{1} \neq 0$ and $A_{2}=0$, then $R_{11}=B_{1} / A_{1}$ and $T_{12}=B_{2} / A_{1}$ are the amplitudes of the probability that the incoming wave is returning in the channel 1 and is transmitted into channel 2, respectively. Similarly, if $A_{1}=0$ and $A_{2} \neq 0$ then $R_{22}=B_{2} / A_{2}$ and $T_{21}=B_{1} / A_{2}$ are the amplitudes of the probability that the incoming wave is returning in the channel 2 and is transmitted into channel 1 , respectively. Substituting $A_{1}=0$ or $A_{2}=0$ into the equation

$$
\left(\begin{array}{c}
B_{1} \\
B_{2}
\end{array}\right)=\left(\begin{array}{c}
S_{11} A_{1}+S_{12} A_{2} \\
S_{21} A_{1}+S_{22} A_{2}
\end{array}\right)
$$

we see that the $S$-matrix consists of these transmission and reflection amplitudes, namely,

$$
S(E)=\left(\begin{array}{lll}
R_{11}(E) & , & T_{21}(E) \\
T_{12}(E) & , & R_{22}(E)
\end{array}\right)
$$

In general the energy $E$ is complex and only for real energies the quantities $R_{n n}$ and $T_{n n^{\prime}}$ have the simple physical meaning.

Also note that the $S$-matrix and therefore the observable transmission and reflection amplitudes do not depend on the normalization of the Jost matrices. Indeed, any common factor of $f^{\text {(in) }}$ and $f^{\text {(out) }}$ cancels out in Eq. (46) which supports our suggestion to move away from their fixed normalization.

\subsection{Partial widths}

At the energy points corresponding to bound states, and at complex energies in the resonance region (fourth quadrant) of the $E$-plane the elements of the $S$-matrix have special properties as well. From Eqs. (46) and (40), we see that at every bound state and resonance

$$
E_{\mathrm{res}}=E_{\mathrm{r}}-i \frac{\Gamma}{2}
$$


the $S$-matrix has a pole. If the energy is close to $E_{\text {res }}$ then

$$
S_{n n^{\prime}}(E) \underset{E \rightarrow E_{\mathrm{res}}}{\approx} \operatorname{const}\left(1-\frac{i \sqrt{\Gamma_{n} \Gamma_{n^{\prime}}}}{E-E_{\mathrm{r}}+i \Gamma / 2}\right),
$$

where $\Gamma_{1}$ and $\Gamma_{2}$ are the partial widths such that together they form the total width

$$
\Gamma=\Gamma_{1}+\Gamma_{2}
$$

of the resonance, and the ratios $\Gamma_{1} / \Gamma$ and $\Gamma_{2} / \Gamma$ are the probabilities that the resonance will decay through (or can be excited from) the first or second channels, respectively. As it follows from Eq. (50), the partial widths $\Gamma_{n}$ can be found as the limits

$$
\Gamma_{n}=\lim _{E \rightarrow E_{\mathrm{res}}}\left(E-E_{\mathrm{res}}\right) S_{n n}(E) .
$$

However, in numerical calculations, finding a limit of a singular functions is not an easy task. A way to avoid this difficulty was suggested by Masui et al. in Ref. [54]. From Eq. (50) it is clear that

$$
\lim _{E \rightarrow E_{\mathrm{res}}}\left|\frac{S_{11}(E)}{S_{22}(E)}\right|=\frac{\Gamma_{1}}{\Gamma_{2}} .
$$

Therefore the partial widths can be found with the help of Eqs. (51) and (52), if $\Gamma$ is known and if there is a procedure for the $S$-matrix calculation at complex energies.

We can significantly simplify the task of finding the limit (52) by using Eq. (46). Indeed, both $S_{11}$ and $S_{22}$ have singularities at $E_{\text {res }}$ because of vanishing determinant of $f^{\text {(in) }}$, which

cancels out in their ratio. Making explicit inversion of the $2 \times 2$ matrix $f^{(\text {in) }}$ and using Eq. (46), we obtain

$$
\frac{\Gamma_{1}}{\Gamma_{2}}=\left|\frac{f_{11}^{\text {(out) }} f_{22}^{(\text {in })}-f_{12}^{(\text {out })} f_{21}^{(\text {in })}}{f_{22}^{\text {(out) }} f_{11}^{(\text {in })}-f_{21}^{\text {(out) }} f_{12}^{(\text {in })}}\right|_{E=E_{\text {res }}},
$$

where no singularities are present.

\section{Complex energies}

The differential equations (31) enable us to obtain a complete solution of the two-channel (generally, $N$-channel) problem at any energy of physical interest. Their advantage over the corresponding Schrödinger equation becomes especially evident when we consider complex values of the energy. 


\subsection{Riemann surface}

Since the functions $\mathcal{F}$ (in/out) $(E, r)$, and therefore the Jost matrices, depend on the energy $E$ via the channel momenta $k_{1}=\sqrt{\left(2 \mu_{1} / \hbar^{2}\right)\left(E-E_{1}\right)}$ and $k_{2}=\sqrt{\left(2 \mu_{2} / \hbar^{2}\right)\left(E-E_{2}\right)}$, there are two square-root branching points for these matrices in the energy plane : $E=E_{1}$ and $E=E_{2}$. This means that if we make two full circles around either of these points then matrix elements of $f^{(\text {in/out })}(E)$ return to the same values from which the circling was started (one full circle is not enough). This is because the channel momentum

$$
k_{n}=\sqrt{\left(2 \mu_{n} / \hbar^{2}\right)\left|E-E_{n}\right| e^{i \chi_{n}}}=\sqrt{\left(2 \mu_{n} / \hbar^{2}\right)\left|E-E_{n}\right|} e^{i \chi_{n} / 2}
$$

comes to its initial value when $\chi_{n} \rightarrow \chi_{n}+4 \pi$, where $\left|E-E_{n}\right|$ and $\chi_{n}$ are the polar coordinates of the point $E$ on the energy-plane relative to the branching point $E_{n}$, i.e.

$$
E=E_{n}+\left|E-E_{n}\right| e^{i \chi_{n}} .
$$

In other words, elements of the Jost matrix have two different values at each point $E$ on this circle.

In order to make the Jost matrix a single-valued function of $E$, we can assume (as is usual in the complex analysis) that the complex energy forms the so-called Riemann surface consisting of several parallel sheets. When doing the first circle around a branching point, we are moving on the first sheet and then continue on the second one until coming back to the first sheet after completing the full two circles. Such continuous transition from one sheet to another is possible if we make a cut from the branching point to infinity, and connect opposite rims of the cuts on the two sheets (see Fig. 1).

As is usual in the scattering theory, we make straight line cuts from both branching points to infinity along the positive real axis. Each of the two sheets related by the first branching point, is further branched at the second branching point. Therefore the full Riemann surface consists of four parallel sheets. We can reach any of these sheets by making an appropriate number of circles around the first and the second branching points.

The physical energy (at which the scattering takes place) is on the positive real axis. We choose the cuts and their interconnections in such a way that the physical scattering energies lie on the upper rims of the both cuts. Starting from these physical energies and moving in the anti-clockwise direction around all branching points, we cover the socalled physical sheet of the energy plane. According to Eq. (54) the channel momenta corresponding to this sheet, have positive imaginary parts. The bound states are also on the physical sheet (this is necessary to guarantee the exponential attenuation of their wave functions). The resonances, however, correspond to zeros of the Jost-matrix determinant 
at the momenta with negative imaginary parts and therefore lie on the unphysical sheet of the Riemann surface. The resonances that are able to decay into both channels must have $\operatorname{Im}\left(k_{1}\right)<0$ and $\operatorname{Im}\left(k_{2}\right)<0$. This means that they lie on the sheet which is unphysical with respect to the both branching points.

In practical calculations, we can choose point on one of the four Riemann sheets by selecting appropriate signs in front of the square roots for $k_{1}$ and $k_{2}$, i.e. appropriate signs of their imaginary parts. These signs must be such that the imaginary part of the momentum is positive for a closed channel and negative for an open one. This corresponds to the choice between the physical and unphysical sheets of the Riemann $E$-surface and ensures correct asymptotic behavior of the wave function.

\subsection{Complex rotation}

Similarly to the single-channel case (see Refs. [47, 48]), the scheme described in previous sections, can be easily implemented only for bound and scattering states. In the resonance domain of the complex $E$-plane (below the real axis) the Riccati-Hankel function $h_{\ell}^{(+)}(k r)$, as can be seen from its asymptotics

$$
h_{\ell}^{( \pm)}(z) \underset{|z| \rightarrow \infty}{\longrightarrow} \mp i \exp \{ \pm i[z-\ell \pi / 2]\}
$$

diverges when $r \rightarrow \infty$. As a result the right hand side of the first matrix equation of the set (31) diverges and hence the limit (35) cannot be calculated. It should be emphasized that this fact does not mean that this limit does not exist. It do exist, but simply moving along the real $r$-axis, we cannot reach it. It is easy to see, that

$$
\text { if } \operatorname{Im} k r>0 \Longrightarrow h_{\ell}^{(+)}(k r) \underset{|k r| \rightarrow 0}{\longrightarrow} 0
$$

When $\operatorname{Im} k r=0$ this function remains finite (oscillates) at large $r$. The condition $\operatorname{Im} k_{n} r>$ 0 for asymptotic vanishing of the function $h_{\ell}^{(+)}\left(\eta_{n}, k_{n} r\right)$ involves the imaginary part of the product $k_{n} r$ but not of the momentum alone. This offers an elegant way to extend the domain of the $E$-plane where the limit (35) can be calculated, to practically whole $E$ plane. Indeed, if, for example, $\operatorname{Im} k_{n} r$ is negative we can always make it positive by using complex values of $r$. This of course requires that the potential is defined for complex $r$ and tends to zero when $|r| \rightarrow \infty$ at least in certain sector of the complex $r$-plane. We assume that it vanishes faster than $r^{-2}$ when $r \rightarrow \infty$ along any line

$$
r=z \exp (i \theta), \quad z \geq 0
$$


for the rotation angle $\theta$ in the interval $0 \leq|\theta| \leq \theta_{\max }<\pi / 2$. Then, firstly, according to the existence theorem, the solutions $\phi_{n n^{\prime}}(E, r)$ of the Schrödinger equation are holomorphic functions of $r$ within the corresponding domain of the $r$-plane and, secondly, they have the asymptotic behavior of the type (37) along any line (158). Moreover, the coefficients $f^{\text {(in/out) }}(E)$ in such asymptotics are the same for all choices of the rotation angle because they do not depend on $r$.

Similarly to Eq. (57), we have

$$
\text { if } \operatorname{Im} k r<0 \Longrightarrow h_{\ell}^{(-)}(k r) \underset{|k r| \rightarrow 0}{\longrightarrow} 0
$$

Therefore, the limiting values $\mathcal{F}^{\text {(in/out) }}(E, \infty)$ can be found as the corresponding coefficients in the asymptotics of the functions $\mathcal{F}^{\text {(in/out })}\left(E, z e^{i \theta}\right)$. It can be shown that with $\operatorname{Im} k r \geq 0$ the right hand side of the first equation of the set (31) at large distances tends to zero, which means vanishing of the derivative on the left hand side, i.e. that $\mathcal{F}^{\text {(in) }}$ becomes constant (reaches its limit). Similarly, it can be shown that the limit (36) can be reached if $\operatorname{Im} k r \leq 0$. Both limits, i.e. $f^{(\text {in })}(E)$ and $f^{(\text {out })}(E)$ can be found simultaneously only when $\operatorname{Im} k r=0$ and at all spectral points. The line $\operatorname{Im} k r=0$ therefore serves as the dividing line that separates two domains of the complex energy plane. The Jost matrix $f^{(\text {in) }}(E)$ can be calculated above this line, while $f^{(\text {out })}(E)$ below it.

By considering complex $r$, we actually do the analytic continuation of $f^{\text {(in/out) }}(E)$ across the dividing lines (the unitary cuts) to the domains where Eqs. (31) do not give finite values for these matrices. The dividing lines can be turned down wards to expose the resonance spectral points, by rotating $r$ as given by Eq. (58). Indeed, if $\chi_{n}$ is the polar angle parametrizing the position of a point on the $E$-plane relative to the branching point $E_{n}$, then by choosing large enough $\theta$ we can make $\operatorname{Im} k_{n} r$,

$$
\operatorname{Im} k_{n} r=\operatorname{Im}\left(\left|k_{n}\right| z e^{i\left(\theta+\chi_{n} / 2\right)}\right)=\left|k_{l}\right| z \sin \left(\theta+\chi_{n} / 2\right)
$$

positive even when $\chi_{n}$ is negative (when the point $E$ is below the cut, i.e. on the unphysical sheet) and vice versa. From the last equation is clear that when $\theta>0$ both dividing lines are turned down by $2 \theta$ (see Fig. 2).

The fact that $f^{\text {(in) }}(E)$ and $f^{\text {(out) }}(E)$ can be found simultaneously only on the lines corresponding to the unitary cuts and at the spectral points, does not pose a problem. Indeed, for bound states $\operatorname{Im} k r>0$ and we can locate them using Eq. (40) without the rotation. As soon as a bound state has been located at a spectral point $E_{b}$, we can calculate both $\mathcal{F}^{( \pm)}\left(E_{b}, r\right)$ at this point and thus obtain the wave function. The scattering takes place exactly on the real axis that coincides with the unitary cut where both $f^{(\mathrm{in})}(E)$ and $f^{\text {(out) }}(E)$ can be calculated without rotation. To locate resonances, we turn the cuts 
down opening the unphysical energy sheets, and again use Eq. (40) which involves only $f^{(i n)}(E)$. If we need to obtain the partial widths (which requires the knowledge of the $f^{(\text {out })}(E)$ as well), we have to repeat the integration of the differential equations at the resonance energy with such rotation angle that the resonance in question is below the cut. It should be also mentioned that the border separating the two domains of the complex $E$-plane is a line only in the case of potentials slowly vanishing at infinity (for example, as $\left.1 / r^{n}\right)$. If, however, the potential decays exponentially, then $f^{(i n)}(E)$ can be found not only above the dividing line but also within a band below this line and $f^{(\text {out })}(E)$ within a symmetrical band above the line (see Ref. [50]). The faster the potential decays the wider this band is.

\subsection{Integration path}

As was shown above, when solving the differential equations (31), we have to move from the origin to infinity (actually to a large $|r|=R$ ) along the ray (58) with generally nonzero $\theta$. In numerical calculations, however, this straightforward approach is not always practical. As was mentioned earlier, in the immediate vicinity of the point $r=0$, the singularities of the Riccati-Hankel functions cancel each other in accordance with Eq. (32). This may make Eqs. (31) numerically unstable near $r=0$. Such an instability is further aggravated when the angular momentum $\ell$ becomes large.

This problem can be easily circumvented in two ways. One way is to solve the matrix Schrödinger equation (14) on an interval from a sufficiently small $r=r_{\text {min }}$ to an intermediate point $r=b$. Having thus calculated the matrices $\phi(E, b)$ and $\phi^{\prime}(E, b)$, we can obtain the values $\mathcal{F}^{\text {(in/out) }}(E, b)$ using the relations

$$
\mathcal{F}^{\text {(in/out) }}(E, r)= \pm \frac{1}{2 i} K^{-1}\left[W_{J}^{(\text {out } / \text { in })}(E, r) \phi(E, r)-W_{J}^{(\text {out } / \text { in })}(E, r) \phi^{\prime}(E, r)\right]
$$

which follow from Eqs. (20) and (22). Then we can turn to the ray (58) along the path shown in Fig. 3, and safely proceed with Eqs. (31) to a sufficiently large $z=R$, which will serve as the "infinity".

Another way is to rearrange Eqs. (31) on the interval $\left[r_{\min }, b\right]$ in order to avoid the singularity cancellations. Since $\left(h_{\ell}^{(+)}+h_{\ell}^{(-)}\right) / 2=j_{\ell}$ and $\left(h_{\ell}^{(+)}-h_{\ell}^{(-)}\right) / 2 i=n_{\ell}$, where $n_{\ell}$ is the Riccati-Neumann function (which is singular at $r=0$ ), we may introduce a new pair of matrices

$$
\mathcal{A}(E, r)=\mathcal{F}^{\text {(in) }}(E, r)+\mathcal{F}^{\text {(out) }}(E, r)
$$

and

$$
\mathcal{B}(E, r)=i\left[\mathcal{F}^{(\text {in })}(E, r)-\mathcal{F}^{(\text {out })}(E, r)\right]
$$


which transform the ansatz (20) into the form

$$
\phi(E, r)=\mathcal{J}_{\ell}(E, r) \mathcal{A}(E, r)-\mathcal{N}_{\ell}(E, r) \mathcal{B}(E, r)
$$

where

$$
\mathcal{J}_{\ell}(E, r)=\left(\begin{array}{cc}
j_{\ell}\left(k_{1} r\right) & 0 \\
0 & j_{\ell}\left(k_{2} r\right)
\end{array}\right)
$$

and

$$
\mathcal{N}_{\ell}(E, r)=\left(\begin{array}{cc}
n_{\ell}\left(k_{1} r\right) & 0 \\
0 & n_{\ell}\left(k_{2} r\right)
\end{array}\right) .
$$

The corresponding linear combination of Eqs. (31) gives their alternative form

$$
\left\{\begin{array}{l}
\frac{d}{d r} \mathcal{A}=-K^{-1} \mathcal{N}_{\ell} V\left[\mathcal{J}_{\ell} \mathcal{A}-\mathcal{N}_{\ell} \mathcal{B}\right] \\
\frac{d}{d r} \mathcal{B}=-K^{-1} \mathcal{J}_{\ell} V\left[\mathcal{J}_{\ell} \mathcal{A}-\mathcal{N}_{\ell} \mathcal{B}\right]
\end{array}\right.
$$

with the boundary conditions that follow from (34)

$$
\lim _{r \rightarrow 0} \mathcal{A}_{n n^{\prime}}(E, r)=2 \delta_{n n^{\prime}}, \quad \lim _{r \rightarrow 0} \mathcal{B}_{n n^{\prime}}(E, r)=0
$$

The representation of $\phi(E, r)$ in terms of $\mathcal{A}, \mathcal{B}$ and $\mathcal{F}^{\text {(in/out) }}$ is equivalent. However, from a practical point of view, near the origin it is more convenient to use Eqs. (66) which do not have the problem of singularity cancellation and thus are numerically stable. At large distances, we have to solve Eqs. (31) in order to obtain the Jost matrices ( $\mathcal{A}$ and $\mathcal{B}$ involving both $\mathcal{F}^{\text {(in/out) }}$, generally do not converge with any rotation angle). Thus, we can follow the same path shown in Fig. 3, making transformation from $\{\mathcal{A}, \mathcal{B}\}$ to $\left\{\mathcal{F}^{\text {(in) }}, \mathcal{F}^{\text {(out) }}\right\}$ at $r=b$.

In principle, from the very beginning when solving either the Schrödinger equation or Eqs. (66), we could move along the ray (58). This however could be a source of numerical instability of another kind. If the potential has exponential functions, it is oscillating with complex $r$. Moving along the real axis allows us to avoid this problem. Moreover, the potential may be given in an analytical form only for large $r$ while in the inner region it is known in the form of a table along the real axis. 


\section{Numerical example}

In order to demonstrate the efficiency and accuracy of the proposed method, we apply it here to a particular two-channel problem. Another purpose of the calculations reported further down, is to learn how individual resonances contribute to observable scattering cross sections.

\subsection{Potential}

As a testing ground, we chose the model proposed by Noro And Taylor [59] and used in many other publications since then. Their two-channel potential

$$
U(r)=\left(\begin{array}{rr}
-1.0 & -7.5 \\
-7.5 & 7.5
\end{array}\right) r^{2} e^{-r}
$$

is of a short range and apparently obeys the condition (77). The units in this model are chosen in such a way that $\mu_{1}=\mu_{2}=\hbar c=1$. In the tables and figures given in the subsequent Sections, the units for the energies and cross sections are therefore not indicated.

The threshold energies are $E_{1}=0$ and $E_{2}=0.1$. The potential (68) has an attractive well in the lower channel, a barrier in the upper channel, and rather strong coupling between the two channels. This means that it generates a rich spectrum and represents a real challenge to any method that is designed to analyze the roles played by individual resonances in the whole picture of the scattering process.

All the calculations presented here, were done for $\ell=0$. This is not because our method has some limitations or needs simplifications. It can handle any value of the angular momentum (even complex). Since however this is an artificial model and there are no partial waves that would present a special physical interest, we chose the lowest one. Moreover, to the best of our knowledge, in all other publications only the $S$-wave scattering was considered and we therefore have to do the same to be able to compare some results.

\subsection{Spectral points and cross sections}

Using the Runge-Kutta-Fehlberg method [60], we solved Eqs. (66) from $r_{\min }=0.0001$ to $b=1$ along the real axis. Then with the same numerical method, we solved Eqs. (31) from $r=b$ to $|r|=30$ along the path shown in Fig. 3. For searching the bound states and calculating the $S$-matrix for real energies, the rotation angle $\theta$ was taken to be zero. When the resonances were located and the $S$-matrix was calculated in the complex 
$E$-plane, we used appropriate values for this angle (up to $0.4 \pi$ ) that turned the unitary cuts (see. Fig. 2) far enough to open necessary domains of the complex plane.

The spectral points nearest to the origin of the E-plane are given in Table 1 where the corresponding results from some other publications are presented for the sake of comparison. The $S$-matrix poles corresponding to these spectral points are shown in Fig. 4. The potential (68) supports four bound states and generates a string of nine resonances at energies above the second threshold. All other spectral points are subthreshold resonances and lie at negative energies.

The two lowest bound states lie below the minimum of the attractive potential in the first channel. This shows that the system under consideration is essentially diabatic as one should expect with strong cross-channel coupling in the potential (68).

The $S$-wave scattering cross sections for the transitions from channel $n$ to channel $n^{\prime}$,

$$
\sigma\left(n \rightarrow n^{\prime}\right)=\frac{\pi}{k_{n}}\left|\delta_{n^{\prime} n}-S_{n^{\prime} n}\right|^{2},
$$

presented in Figs. 5, 6, and 7, show several bumps and peaks. Our traditional understanding of quantum scattering, preoccupied with the Breit-Wigner picture, would push us towards associating these cross section irregularities with individual resonance poles of the $S$-matrix. Let us not jump to such conclusions too hastily. Having analyzed how the cross section is built up out of individual contributions from the resonances, in the subsequent sections we will see that the $\sigma\left(n \rightarrow n^{\prime}\right)$ curves are the results of rather complicated interplay of all the poles and therefore a Breit-Wigner parametrization of them would be naive and misleading.

\subsection{Mittag-Leffler expansion}

The Mittag-Leffler theorem [61] offers a way to decompose the $S$-matrix in a sum of terms representing its poles and the background. The theorem itself is rather general. In plain words, it says that a meromorphic function can be expanded in a sum of an entire function and a series of its principal parts at all the poles. What we actually need is more simple. Leaving the mathematical subtleties, we want to expand the $S$-matrix in a sum of few singular terms (poles) and something that takes into account everything else (background term). This can be done using the Cauchy integral formula. Indeed, consider a contour shown in Fig. 8, which encloses $N$ poles of the $S$-matrix. Then the Cauchy formula reads

$$
\oint \frac{S(\zeta)}{\zeta-E} d \zeta=2 \pi i S(E)+2 \pi i \sum_{j=1}^{N} \frac{\operatorname{Res}\left[S\left(E_{j}\right)\right]}{E_{j}-E}
$$


where $E$ is a point inside the contour and $E_{j}$ are the resonance energies. Therefore in the desired expansion,

$$
S(E)=\sum_{j=1}^{N} \frac{\operatorname{Res}\left[S\left(E_{j}\right)\right]}{E-E_{j}}+\frac{1}{2 \pi i} \oint \frac{S(\zeta)}{\zeta-E} d \zeta
$$

the $N$ selected poles are represented explicitly while the rest of them as well as the background (the Mittag-Leffler's entire function) are taken into account via the contour integral. In order to understand what role a particular resonance plays in the scattering process, we will simply omit the corresponding term from the sum (171) and analyze the consequences. In a sense, the expansion (71) is similar to that of Ref. [41] where the spectral-density function $m^{+}(\lambda)$ was presented as a sum over the resonances and the background term. It should be noted, however, that Eq. (71) is not an approximation but an exact expansion when all $N$ poles enclosed by the contour are taken into account. To calculate the $S$-matrix via Eq. (71), we need to know its residues at the poles and to be able to calculate the integral for a given energy $E$. The residues can be found by numerical differentiation of the Jost matrix determinant. Indeed, according to Eq. (46)

$$
S=f^{\text {(out) }}\left(\begin{array}{cc}
f_{22}^{(\text {in })} & -f_{12}^{(\text {in })} \\
-f_{21}^{(\text {in })} & f_{11}^{(\text {in })}
\end{array}\right) \frac{1}{\operatorname{det} f^{(\text {in })}},
$$

where the poles are caused by simple zeros of $\operatorname{det} f^{(\mathrm{in})}(E)$. Therefore

$$
\operatorname{Res}[S(E)]=f^{(\text {out })}(E)\left(\begin{array}{cc}
f_{22}^{(\text {in })}(E) & -f_{12}^{(\text {in })}(E) \\
-f_{21}^{(\text {in })}(E) & f_{11}^{(\text {in })}(E)
\end{array}\right)\left[\frac{d}{d E} \operatorname{det} f^{(\text {in })}(E)\right]^{-1}
$$

with the derivative numerically obtained within the central difference approximation

$$
\frac{d}{d E} \operatorname{det} f^{(\mathrm{in})}(E) \approx \frac{\operatorname{det} f^{(\mathrm{in})}(E+\epsilon)-\operatorname{det} f^{(\mathrm{in})}(E-\epsilon)}{2 \epsilon} .
$$

In the calculations, we used $\epsilon=10^{-6}$ which gave the accuracy of at least 5 digits. This was sufficient for the purpose of visual comparing the curves when analyzing the contribution to the total cross section from different $S$-matrix poles.

Thus calculated residues of the $S$-matrix are given in Table 2 and plotted in Fig. 9. It is interesting to notice that they follow regular pattern in the form of anti-clockwise spiral. The reason for growth of the spiral radius is that according to Eq. (50)

$$
\operatorname{Res}\left[S_{n n^{\prime}}(E)\right] \sim \sqrt{\Gamma_{n} \Gamma_{n^{\prime}}}
$$


and the widths become larger every time we go to the next resonance. The reason for the anti-clockwise turning of the corresponding phase factor, however, remains unclear. As is seen from Fig. 10, the phases of the residues show the tendency to converge to a constant angle, which means that for the far away resonances the points on the spiral become closer and closer to each other.

The contour integral of Eq. (71), enclosing nine $(N=9)$ resonance points (see Fig. 8), was calculated using 175 points of the Gaussian quadrature formula on each of the three line segments that form the contour. With this number of quadrature points we achieved four digit accuracy which was more than sufficient for our purpose of visual comparing the cross section curves. The vertices of the triangle contour $(0.5,0.5),(0.5,-30.0)$, and $(20.1,0.5)$ were chosen in such a way that both branching points are outside of it and with $\theta \geq 0.25 \pi$ the contour does not cross the unitary cuts.

A question may arise: Why did we not include the bound state poles in the contour? There are two reasons for this. Firstly, the present work was motivated (as we explained in the Introduction) by the controversy in associating the irregularities of the chemical reaction cross sections with intermediate resonant states. Therefore, our goal was to develop a technique for examining the contributions of the resonance poles, not the bound states. Secondly, a contour enclosing the bound states, would require calculating the $S$-matrix via Eq. (46) at the points around them, i.e. with $\operatorname{Im} k_{n}>0$. At such points, however, the limit (36) does not exist. In order to reach these points, we would need a negative rotation angle with $\theta<-\pi / 2$ which is not possible. There is a way to circumvent this difficulty by expanding the solutions $\mathcal{F}^{\text {(in/out) }}$ in series near $E=0$ (see Ref. [46]), but this would require a separate publication. It should be emphasized that the inclusion of only nine poles in the contour is not an approximation. The Cauchy theorem (70) is exact, provided that all enclosed poles are taken into account. All the other poles are taken care of by the contour integral.

\subsection{Analysis of the cross sections}

Before starting the analysis, we tested the numerical procedure by comparing the cross sections directly obtained from Eqs. (31) at real energies with $\theta=0$ and the corresponding cross sections calculated from the expansion (71). The same $\sigma\left(n \rightarrow n^{\prime}\right)$ curves, namely, shown in Figs. 5, 6, and 7, were obtained in both cases (within four digit accuracy, which is visually indistinguishable). Having thus established that the sum (71) reproduces the cross sections correctly, we started to investigate the importance of its terms by excluding them from the sum and calculating the resulting cross sections.

First of all, we omitted all the nine poles. To our surprise, the remaining integral part of the expansion (71) did not turn out to be a smooth function. Contrary to our expectation, 
at low energies it gives almost the same structure of peaks and deeps as they are in the exact cross sections (see Figs. 11, 12, and 13). This means that these peaks are of a nonresonance origin. They are either the threshold irregularities (known in scattering theory as threshold cusps [45, 62]), or the influence of some other $S$-matrix poles (especially the four bound states that are close to the point $E=0$ ).

In an attempt to understand what role each individual resonance plays in the scattering process, we excluded them one at a time from the sum (71). This procedure can give us some indication and hints. In Figs. 14, 15, and 16 the cross sections $\sigma(1 \rightarrow 1), \sigma(1 \rightarrow 2)$, and $\sigma(2 \rightarrow 2)$ thus obtained are shown for eight different choices of the resonance to be excluded.

These figures do not show the effect of the exclusion of the first resonance because this effect is very simple. When the first resonance is omitted, the narrow deep in $\sigma(1 \rightarrow 2)$ and peak in $\sigma(2 \rightarrow 2)$ at $E=4.768$ disappear while the other parts of the cross section curves remain unchanged. As far as the elastic cross section $\sigma(1 \rightarrow 1)$ is concerned, the resonant cusp at $E=4.768$ (see insert on Fig. 5) is so weak that it is hardly visible and therefore the exclusion of the first resonance pole from the sum (71) is practically unnoticeable. The reason for that can be found in Table 2. The $S_{11}$ element of the $S$ matrix has extremely small residue at this pole. As a result the corresponding term in the sum (71) for real $E$ is always negligible. Being used to the Breit-Wigner picture, we expected to have a sharp energy dependence at around $E \sim 4.8$ in all channels. To our surprise, this turned out to be not the case because of smallness of $\operatorname{Res}\left(S_{11}\right)$.

Apart from the first resonance $(E=4.768)$, the only place where we can see a clear manifestation of a resonance through a distinct peak is the top left corner of Fig. 15, where one of the peaks in the inelastic cross section $\sigma(1 \rightarrow 2)$ disappears together with the second resonance pole while the rest of the curve retains its shape. As far as the elastic scattering in the channels 1 and 2 is concerned (see Figs. 14 and 16), the only information we can deduce from the curves is that the second and the third resonances are responsible for the bumps between $E=5$ and $E=10$, the far resonances do scaling of the curves, and no resonances manifest themselves through distinct peaks (except for the first one, of course). This is from a general physical view understandable in the sense that the fourth and higher resonances are so wide that they, when excited, affect a rather wide energy region.

In general, the cross section analysis based on the expansion (71), is not straight forward. This is not surprising because the contributions of the terms of this expansion into the cross section are not linear. Indeed, on the right hand side of Eq. (69) in addition to squares of these terms there are many interference products whose contributions depend on relative phases of the terms. Therefore the full cross section is a result of complicated interplay of all resonances and the background term. The analysis of the sum (71) itself, 
i.e. examining the contributions of the resonances into the $S$-matrix, could shed some light on the role of individual resonances in the scattering picture. The most convenient way of doing this is the analysis of the Argand plots which is done next.

\subsection{Argand plots}

The Argand plots are widely used to depict complex valued functions depending on a real parameter. For each value of this parameter, the function value corresponds to a point in the complex plane. If the function is continuous, the set of such points forms a curve.

In the scattering theory, the term Argand plot has a specific meaning, namely, the curve in the complex plane, along which the $S$-matrix element moves when the (real) collision energy increases. Traditionally the Argand plots were mainly used in particle physics. There are however many examples of their application in atomic and molecular physics as well [63, 64].

It can be shown (see, for example, Refs. 63] and 65]) that in the absence of resonances the phases (arguments) of elements of the $S$-matrix monotonously decrease with the increasing energy of collision. Therefore these matrix elements as functions of real energy move along circular trajectories in the clockwise direction on the Argand plot. Contrary to that, a resonance causes the scattering phase shift to increase by $\pi$, which means an increase of the $S$-matrix phase by $2 \pi$ (i.e. full anti-clockwise circle). This is, of course, an idealized picture for an isolated resonance with weak influence of the background scattering. In a vicinity of a resonance, the $S$-matrix can always be split in two terms: The background and resonance terms, with decreasing and increasing phases, respectively. Their interplay may cause the curvature of the Argand trajectory to change. If a resonance is strong, the curvature changes so much as making the point on the curve to move along an arc in the anti-clockwise direction. With a weak resonance, the curve changes its curvature only slightly.

In Figs. 17, 18, and 19 the Argand plots for the $S$-matrix generated by the potential (68) are shown in the energy interval $0.5 \leq E \leq 20$. The dots on the curves mark the integer values of the energy, namely, $E=1,2,3, \ldots$ As is seen, all three matrix elements have three anti-clockwise arcs in the intervals $4.765<E<4.775,6<E<9$, and $9<E<20$. This means that the corresponding peaks at $E=4.768$ and bumps seen between $E \sim 6$ and $E \sim 20$ in Figs. 5, 6, and 7, are of the resonance origin, despite the fact that not all of them are high or sharp. The only question remains: Which poles are responsible for these resonances?

To answer this question, we make use of the expansion (71). There is no problem of interference here because, in contrast to the cross section, the contribution of each pole to the $S$-matrix is linear. Therefore, excluding them one at a time, we can find out which 
poles the two anti-clockwise arcs of the Argand plots are associated with.

First of all, as was mentioned before, the first resonance pole does not contribute to anything when $E$ moves away from the point $E=4.768$. This is because the denominator of the corresponding term in the sum (71) becomes much lager than the residue. The omission of the first resonance term results therefore in the disappearance of the first anticlockwise circles $(4.765<E<4.775)$ in Figs. 17, 18, and 19, The rest of these figures remain unaffected. Contrary to that, the second pole makes a noticeable contribution everywhere. In Fig. 20 the Argand plots for the three elements of the $S$-matrix are shown when this pole is excluded from the sum (71). It is seen that in all the channels there is no second anti-clockwise arc between $E \sim 6$ and $E \sim 9$ as compared with Figs. 17, 18, and 19. We therefore can conclude that the anti-clockwise arc in the interval $6<E<9$ is associated with the second pole and the bumps in this energy interval on the cross sections shown in Figs. 5, 6, and 7, are the manifestation of the second resonance pole, namely, at $E=7.241200-(i / 2) 1.511912$.

It is seen that with the exclusion of the second resonance pole, we still have anti-clockwise arcs for $S_{11}(E)$, starting from $E \sim 9$, and for $S_{21}(E)$ and $S_{22}(E)$, starting from $E \sim 6$ and extending to $E \sim 20$. This means that one or several other resonance poles are responsible for their formation.

In order to examine this, we restore the second pole and exclude the third resonance pole from the expansion (71). The resulting Argand plots are shown in Fig. 21. As was expected, the second anti-clockwise arc $(6<E<9)$ came back together with the second pole. The third arc $(E>9)$ however did not disappear together with the third pole. Therefore the third pole is not fully responsible for this arc and thus for the wide bump on the cross sections beyond $E \sim 9$.

This bump, which is seen in all three Figs. 5, 6, and 7, is a collective effect of several resonances, namely, the third, fourth, and so on. With the presence of the third pole, the anti-clockwise trajectories for $E>9$ on the Argand plots of $S_{21}(E)$ and $S_{22}(E)$ form closed loops. When this pole is excluded, the curvature of these loops is reduced to the extent that the closed loops become just open arcs. Since they do not disappear entirely, we conclude that the fourth and the subsequent poles also contribute to their formation, although not so much as the third pole does.

In order to convince ourselves that the two bumps on the cross sections between $E \sim 6$ and $E \sim 20$ are of pure resonant nature, we excluded all nine poles from the sum (71), i.e. left only the background integral term. The Argand plots thus obtained are given in Fig. 22. It is seen that they show no anti-clockwise motion. Only close to $E=20$ the Argand trajectories become flat and perhaps change sign of the curvature. This means that the integral term practically does not contribute to formation of the bumps. It takes into account all the other poles that are outside the integration contour. The Argand plots 
given in Fig. 22, show that these outside poles contribute very lightly at higher energies. The flattening of the curves is the indication of this.

Finally, it is worthwhile to say a few words about the requirement of unitarity which is violated when one analyzes the Argand plots using a truncated Mittag-Leffler expansion. Since the physical $S$-matrix is unitary, its matrix elements cannot be outside the unitary circle (circle of unit radius) on the Argand plots. This is indeed the case with the curves given in Figs. 17, 18, and 19, However when we omit some terms in the expansion (71), the unitarity is violated. As a result the Argand trajectories are not bound to the unitary circle anymore (see Figs. 20 and 21). This should not be an obstacle. Actually, we are not interested in absolute position of the curve in the complex plane. What we look for are the segments of anti-clockwise motion. In fact, when in our example all nine resonance poles are excluded the unitarity is violated so much that the Argand trajectories shift very far from the origin of the complex plane. This is why in Fig. 22 we do not indicate the coordinate system (which in this case does not give any meaningful information anyway).

\section{Conclusion}

The method presented in this paper, for solving a two-channel problem is based on first order differential equations that are equivalent to the corresponding Schrödinger equation but are more convenient for dealing with the resonant phenomena. The main advantage of this method is that it enables us to directly calculate the Jost matrix for practically any complex value of the energy. The spectral points (bound and resonant states) can therefore be located in a rigorous way as zeros of the Jost matrix determinant.

When calculating the Jost matrix, we solve differential equations and thus at the same time obtain the wave function with the correct asymptotic behaviour that is embedded in the solution from the outset in an analytical form. The method therefore gives a complete solution of the problem in one run. Another advantage of the method used in this paper, is that it offers very accurate way of calculating not only total widths of resonances but their partial widths as well.

Although in this paper we deal with a two-channel problem, all the formulae can be easily generalized to any number of channels. For this, we only need to change the matrix dimensions from 2 to an appropriate number $N$.

Charged particles can also be considered within this method with minor modifications. For this, we need to use the Coulomb functions $F_{\ell}(\eta, k r)$ and $G_{\ell}(\eta, k r)$ instead of the Rikkati-Bessel and Riccati-Neumann functions $j_{\ell}(k r)$ and $n_{\ell}(k r)$, and their combinations $F_{\ell}(\eta, k r) \mp i G_{\ell}(\eta, k r)$ instead of the Riccati-Hankel functions $h_{\ell}^{( \pm)}(k r)$ when constructing the wave functions and deriving the equations. For $\eta \rightarrow 0$ such equations will be 
automatically transformed into the equations given here.

The possibility of calculating the Jost matrix and the $S$-matrix for any complex energy in the resonance domain, enables us not only to locate the $S$-matrix resonance poles but to calculate its residues at these poles. This, in turn, makes it possible to obtain the MittagLeffler type expansion of the $S$-matrix as a sum of the singular terms (representing the resonances) and the background term (contour integral). Such an expansion is very useful in analyzing the contribution of individual resonances into the scattering.

The analysis of the cross section turned out to be less informative than expected. The reason for this is that the cross section being a quadratic function of the $S$-matrix, involves all possible interference terms which smear the contributions from individual resonances and make it difficult to clearly separate them. However, using the Argand plot technique, one can, as demonstrated above, elucidate the origin of the structures in a computed cross section as being the effects of a single isolated resonance, a collective contribution of a set of resonances, or a non-resonant background scattering (which in fact is a collective contribution from all far poles of the $S$-matrix).

The two-channel model by Noro and Taylor, considered in this paper as an example, exhibits these difficulties in an extreme form. This is because its spectrum involves a string of almost completely overlapping resonances. Even in such an extreme case, with the proposed approach, we managed to assign the irregularities of the cross section to the resonances and the background term.

Although this model might look artificial, the spectrum of resonances generated by the Noro and Taylor potential, is similar to the spectra of some real atomic systems. For example, the existence of a string of overlapping resonances that starts with a narrow one, is a feature that characterizes the charge-transfer reaction $N^{3+}+H \rightarrow N^{2+}+H^{+}$ [15, 16, 17. The analysis of the Noro and Taylor model, given in the present paper, could therefore shed some light on the problems associated with realistic systems. Furthermore, with small numerical modifications, the methods presented here can be directly applied to this charge-transfer reaction. The contribution from each resonant eigenstate can then be quantified by using the corresponding $S$-matrix residue and the Argand plots as demonstrated in this paper.

Such an analysis, may eventually, contribute to the understanding of the $F H D$ resonances [7, 10, 11] discussed in the Introduction. Finally, the complex interference between different resonant states in building the observable quantity - the cross section - tells us that it is absolutely necessary to have theoretical tool, like the present, in order to analyze the high-resolution, low-energy atom-atom, atom-ion and atom-molecule collision experiments that eventually may be performed [29]. 


\section{Acknowledgements}

Financial support from the University of South Africa, the National Research Foundation of South Africa (NRF grant number 2067421), as well as from the STINT (Sweden) is greatly appreciated. One of the authors (SAR) is thankful to Stockholm University for its warm hospitality.

\section{References}

[1] R. S. Friedmann, D. G. Truhlar, Chem. Phys. Lett. 183, 539 (1991).

[2] F. Fernández-Alonso and R. N. Zare, Ann. Rev. Phys. Chem. 53, 67 (2002).

[3] N. Bohr, Nature 137, 344 (1937).

[4] E. G. Bilpuch, In: "Isobaric Spin in Nuclear Physics", J. D. Fox and D. Robson, eds., Academic Press N. Y., 1966, page 235.

[5] R. D. Levine and S.-F. Wu, Chem. Phys. Lett. 11, 557 (1971).

[6] G. C. Schatz and A. Kuppermann, J. Chem. Phys. 59, 964 (1973).

[7] G. C. Schatz, Science 288, 1599 (2000).

[8] D. M. Neumark, A. M. Wodtke, G. N. Robinson, C. C. Hayden, K. Shobatake, R. K. Sparks, T. P. Schafer, and Y. T. Lee, J. Chem. Phys. 82, 3067 (1985).

[9] I. M. Waller, T. Kitsopoulos and D. M. Neumark, J. Chem. Phys. 94, 2240 (1990).

[10] R.T. Skodje, D. Skouteris, D. E. Manolopoulos, S.-H. Lee, F. Dong, and K. Liu, J. Chem. Phys. 112, 4536 (2000).

[11] Discussion following the paper : S.-H. Lee, F. Dong and K. Liu, Faraday Discussions, 127, 49 (2004).

[12] R. Newton, "Scattering Theory of Waves and Particles", Second Ed., Springer Verlag, New York, 1982, page 387.

[13] J. R. Taylor, "Scattering Theory", John Wiley \& Sons, New York, 1972, page 238.

[14] M. H. Alexander, D. E. Manolopoulos, H.-J. Werner, J. Chem. Phys. 113, 11084 (2000). 
[15] R. McCarroll and P. Valrion, Proc. 11-th Int. Conf. on Physics of Electronic and Atomic collisions, ( Amsterdam : North-Holland) Invited papers and progress reports, page 453. (1980).

[16] A. Bárány, E. Brändas, N. Elander and M. Rittby, Physica Scripta T3, 233 (1983).

[17] M. Rittby, N.Elander, E. Brändas and A. Bárány, J. Phys. B 17, L677 (1984).

[18] M. Rittby, N. Elander, E. Brändas, In: "Lecture Notes in Physics", 325, Eds. E. Brändas E., N. Elander, Heidelberg: Springer Verlag 1989, p. 129

[19] P. Krylstedt, Ch. Carlsund, N. Elander, J. Phys. B 22, 1051 (1989).

[20] T. Alferova, N. Elander, Int. J. Quantum Chem. 90, 1361 (2002).

[21] T. Alferova, N. Elander, Int. J. Quantum Chem. (2003) (accepted)

[22] N. Shimakura and M. Kimura, Phys. Rev. A, 44, 1659 (1991).

[23] S. Kita, S. Gotoh, T. Hasegawa and N. Shimakura, J. Chem. Phys. 109, 9713 (1998).

[24] S. Suzuki, N. Shimakura, T. Shirai and M. Kimura, J. Phys. B 31, 1741 (1998).

[25] N. Suzuki ,N. Shimakura and H. Kono, Physica Scripta T92, 435 (2001).

[26] M. C. Bacchys-Montabonel and P.Ceyzeriat, Phys. Rev. A 58, 1162 (1998).

[27] C. H. Havener, M. S. Huq, H. F. Krause, P. A. Schulz and R. A. Phaneuf, Phys. Rev. A 39, 1725 (1989).

[28] M. S. Huq, C. C. Havener and R. A. Phaneuf, Phys Rev. A 40, 1811 (1989).

[29] K.-G. Rensfelt, G. Andler, L. Bagge, M. Blom, H. Danared, A. Källberg, S. Leontain, L. Liljeby, P. Löfgren, A. Paál, A. Simonsson and Ö. Skeppstedt, Proceedings of the European Particle Accelerator Conference, Lucerne, Switzerland 2004, page 1425.

[30] G. Breit and E. Wigner, Phys. Rev. 49, 519 (1936).

[31] U. Fano, Phys. Rev. 124, 1866 (1961).

[32] M. Rittby, N. Elander and E. Br`andas, Phys. Rev. A 261804 (1982);

Mol. Phys. 45, 553 (1982).

[33] A. A. Abramov, A. Aslanyan and E. B. Davies, J. Phys. A 3457 (2001). 
[34] D. G. Truhlar, B. C. Garrett, S. J. Klippstein, J. Chem. Phys. 100, 12771 (1996).

[35] S. C. Althorpe, F. Fernández-Alonso, B. D. Bean, J. D. Ayers, A. E. Pomerantz, R. N. Zare, and E. Wrede, Nature 416, 67 (2002).

[36] D. E. Manolopoulos, Nature 419, 266 (2002).

[37] S. A. Harich, D. Dai, C. C. Wang, X. Yang, S. D. Chao, and R. T. Skodje, Nature 419, 281 (2002).

[38] K. J. Laider and M.C. King, J. Chem. Phys. 87, 2657 (1983).

[39] O. I. Tolstikhin, V. N. Ostrovsky, H. Nakamura, Phys. Rev. A 58, 2077 (1998).

[40] T. Alferova, N. Elander and V. Ostrovsky, Few-Body Systems 34, 185 (2004).

[41] E. Engdahl, E. Brändas, M. Rittby, N. Elander, Phys. Rev. A 37, 3777 (1988).

[42] J. Nuttal, H. L. Cohen, Phys. Rev. 188, 1542 (1969).

[43] T. Rescigno, C. W. McCurdy, Phys. Rev. A 37, 624 (1985).

[44] N. Moiseyev, Physics Reports 302, 211 (1998).

[45] A. I. Baz, Ya. B. Zeldovich, A. M. Perelomov, Scattering, Reactions and Decay in Nonrelativistic Quantum Mechanics, Israel Program for Scientific Translations, Jerusalem (1969).

[46] V. V. Pupyshev and S. A. Rakityansky, Z. Phys. A 348, 227 (1994).

[47] S. A. Rakityansky, S. A. Sofianos, and K. Amos, Nuovo Cim. B 111, 363 (1996).

[48] S. A. Sofianos and S. A. Rakityansky, J. Phys. A: Math. Gen. 303725 (1997).

[49] S. A. Sofianos, S. A. Rakityansky, G. P. Vermaak, J. Phys. G 23, 1619 (1997).

[50] S. A. Rakityansky and S. A. Sofianos, J. Phys. A: Math. Gen. 315149 (1998).

[51] S. A. Sofianos, S. A. Rakityansky, S. E. Massen, Phys. Rev. A 50, 337 (1999).

[52] S. A. Rakityansky and S. A. Sofianos, Few-Body Systems Suppl. 10, 93 (1999).

[53] S. E. Massen, S. A. Sofianos, S. A. Rakityansky, S. Oryu, Nucl. Phys., A 654, 597 (1999). 
[54] H. Masui, S. Aoyama, T. Myo, and K. Kato, Prog. Theor. Phys. 102, 1119 (1999).

[55] H. Masui, S. Aoyama, T. Myo, K. Kato, K. Ikeda, Nucl. Phys. A 673, 207 (2000).

[56] H. Masui, C. Kurokawa, and K. Kato, Prog. Theor. Phys. 110, 233 (2003).

[57] S. A. Rakityansky, Phys. Rev. B 68, 195320 (2003).

[58] S. A. Rakityansky, Phys. Rev. B 70, 205323 (2004).

[59] T. Noro and H. S. Taylor, J. Phys. B: Atom. Molec. Phys. 13, L377 (1980).

[60] C. F. Gerald, P. O. Wheatley, Applied Numerical Analysis, 5-th Ed., Addison-Wesley Publishing Company, New York, 1994, pages 406-407.

[61] a book

[62] L. D. Landau, E. M. Lifshitz, Quantum mechanics, Pergamon, Oxford (1965).

[63] A. Kuppermann, In: Potential Energy Surfaces and Dynamics Calculations for Chemical Reactions and Molecular Energy Transfer, Edited by D. G. Truhlar, Plenum Press, New York, 1981, pages 375-420.

[64] F. J. Aoiz, L. Bañares, J. F. Castillo, J. Chem. Phys., 117, 2546, 2002.

[65] R. H. Dalitz, In: Resonances - Models and Phenomena, Lecture Notes in Physics, v. 211, pp. 1-26, Springer-Verlag, Berlin, 1984.

[66] N. Moiseyev and U. Peskin, Phys. Rev. A 42, 255 (1990). 


\begin{tabular}{|r|r|r|r|r|}
\hline$E_{r}$ & $\Gamma$ & $\Gamma_{1}$ & $\Gamma_{2}$ & Ref. \\
\hline-2.314391 & 0 & 0 & 0 & this work \\
\hline-1.310208 & 0 & 0 & 0 & this work \\
\hline-0.537428 & 0 & 0 & 0 & this work \\
\hline-0.065258 & 0 & 0 & 0 & this work \\
\hline 4.768197 & 0.001420 & 0.000051 & 0.001369 & this work \\
4.768197 & 0.001420 & 0.000051 & 0.001369 & {$[54$} \\
4.7682 & 0.001421 & 0.000061 & 0.001360 & {$[59]$} \\
4.7682 & 0.001421 & 0.000051 & 0.001368 & {$[66]$} \\
\hline 7.241200 & 1.511912 & 0.363508 & 1.148404 & this work \\
7.241200 & 1.511912 & 0.363507 & 1.148405 & {$[54]$} \\
\hline 8.171217 & 6.508332 & 1.596520 & 4.911812 & this work \\
8.171216 & 6.508332 & 1.596517 & 4.911814 & {$[54]$} \\
\hline 8.440526 & 12.562984 & 3.186169 & 9.376816 & this work \\
8.440526 & 12.56299 & 3.186167 & 9.376820 & {$[54]$} \\
\hline 8.072643 & 19.145630 & 4.977663 & 14.167967 & this work \\
8.072642 & 19.14563 & 4.977660 & 14.16797 & {$[54]$} \\
\hline 7.123813 & 26.025337 & 6.874350 & 19.150988 & this work \\
7.123813 & 26.02534 & 6.874348 & 19.15099 & {$[54]$} \\
\hline 5.641023 & 33.070140 & 8.816746 & 24.253394 & this work \\
5.641023 & 33.07014 & 8.816744 & 24.25340 & {$[54]$} \\
\hline 3.662702 & 40.194674 & 10.768894 & 29.425779 & this work \\
3.662702 & 40.19467 & 10.76889 & 29.42578 & {$[54]$} \\
\hline 1.220763 & 47.339350 & 12.709379 & 34.629971 & this work \\
1.220763 & 47.33935 & 12.70925 & 34.63010 & {$[54]$} \\
\hline-1.657821 & 54.460303 & 14.624797 & 39.835506 & this work \\
-1.658115 & 54.46087 & 14.62500 & 39.83587 & {$[54]$} \\
\hline-4.949904 & 61.523937 & 16.507476 & 45.016461 & this work \\
-4.950418 & 61.52509 & 16.50735 & 45.01774 & {$[54$} \\
\hline-8.635366 & 68.503722 & 18.352084 & 50.151638 & this work \\
-8.635939 & 68.50621 & 18.35089 & 50.15532 & {$[54]$} \\
\hline-12.696283 & 75.378773 & 20.155213 & 55.223560 & this work \\
\hline-17.117760 & 82.129712 & 21.915313 & 60.214399 & this work \\
\hline
\end{tabular}

Table 1: Spectral points generated by the potential (68) and shown in Fig. 4, 


\begin{tabular}{|c|c|}
\hline$E$ & $\begin{array}{r}\operatorname{Res}\left[S_{11}(E)\right] \\
\operatorname{Res}\left[S_{21}(E)\right] \\
\operatorname{Res}\left[S_{22}(E)\right]\end{array}$ \\
\hline $4.768197-i 0.000710$ & $\begin{aligned}(-0.04757+i 0.50890) & \times 10^{-4} \\
(0.26429-i 0.02943) & \times 10^{-3} \\
(-0.04234-i 0.13016) & \times 10^{-2}\end{aligned}$ \\
\hline $7.241200-i 0.755956$ & $\begin{array}{r}-0.52405-i 0.05745 \\
0.90478+i 0.25589 \\
-1.50601-i 0.71154\end{array}$ \\
\hline $8.171217-i 3.254166$ & $\begin{array}{r}4.17442-i 0.17731 \\
-7.30357-i 0.80750 \\
12.42094+i 3.31244\end{array}$ \\
\hline $8.440526-i 6.281492$ & $\begin{array}{r}-4.97737+i 10.97961 \\
10.99059-i 17.56517 \\
-22.57772+i 27.36750\end{array}$ \\
\hline $8.072643-i 9.572815$ & $\begin{array}{r}-19.17840-i 7.93820 \\
30.61007+i 17.10104 \\
-47.96303-i 34.49489\end{array}$ \\
\hline $7.123813-i 13.012669$ & $\begin{array}{r}0.53670-i 26.81386 \\
-5.46659+i 44.46505 \\
16.42995-i 72.88532\end{array}$ \\
\hline $5.641023-i 16.535070$ & $\begin{array}{r}24.25310-i 16.59884 \\
-42.53890+i 23.84558 \\
73.67460-i 33.28442\end{array}$ \\
\hline $3.662702-i 20.097337$ & $\begin{array}{r}28.88187+i 4.77348 \\
-46.99352-i 11.58529 \\
75.99725+i 24.95204\end{array}$ \\
\hline $1.220763-i 23.669675$ & $\begin{array}{r}19.53089+i 19.50318 \\
-29.97320-i 34.31696 \\
45.62484+i 59.78651\end{array}$ \\
\hline
\end{tabular}

Table 2: Residues of the $S$-matrix elements at the 9 resonance poles enclosed in the contour shown in Fig. 8 . 


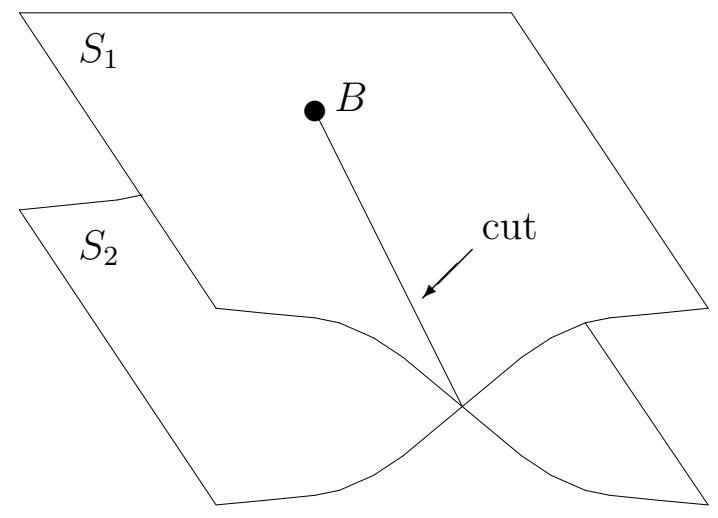

Figure 1: Fragments of the physical $\left(S_{1}\right)$ and unphysical $\left(S_{2}\right)$ sheets of the complex-energy Riemann surface around a branching point $(B)$ that corresponds to a threshold energy $\left(E_{2}\right.$ or $\left.E_{1}\right)$. Transition from $S_{1}$ to $S_{2}$ and back is possible through the unitary cut running from $B$ to infinity.

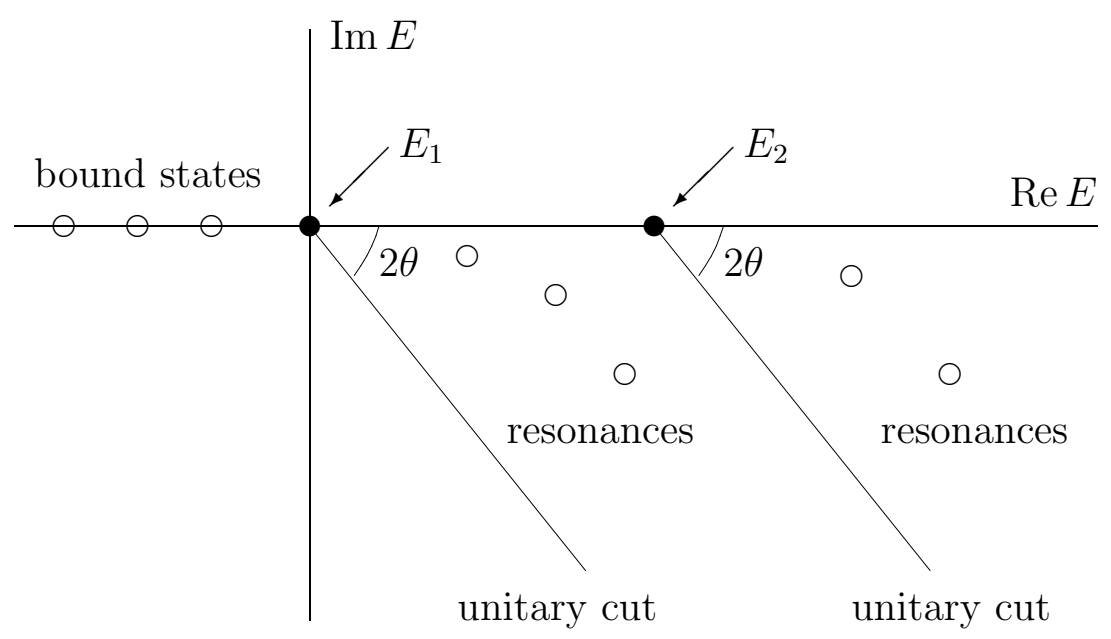

Figure 2: Typical distribution of the bound states and resonances i.e. spectral points (open circles) in the complex energy-plane. It is assumed that $E_{1}<E_{2}$ and the energy is measured relative to the lowest threshold $E_{1}$. The unitary cuts going from the branching points to infinity are also shown. Because of the complex rotation, these cuts are turned into the unphysical sheet by the angle $2 \theta$. 


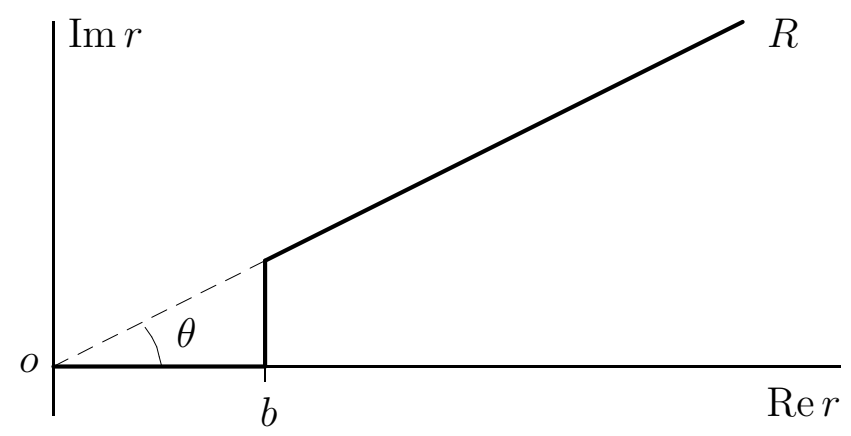

Figure 3: The integration path for solving Eqs. (66) on the segment $o b$, and the Jost function equations (31) on $b R$.

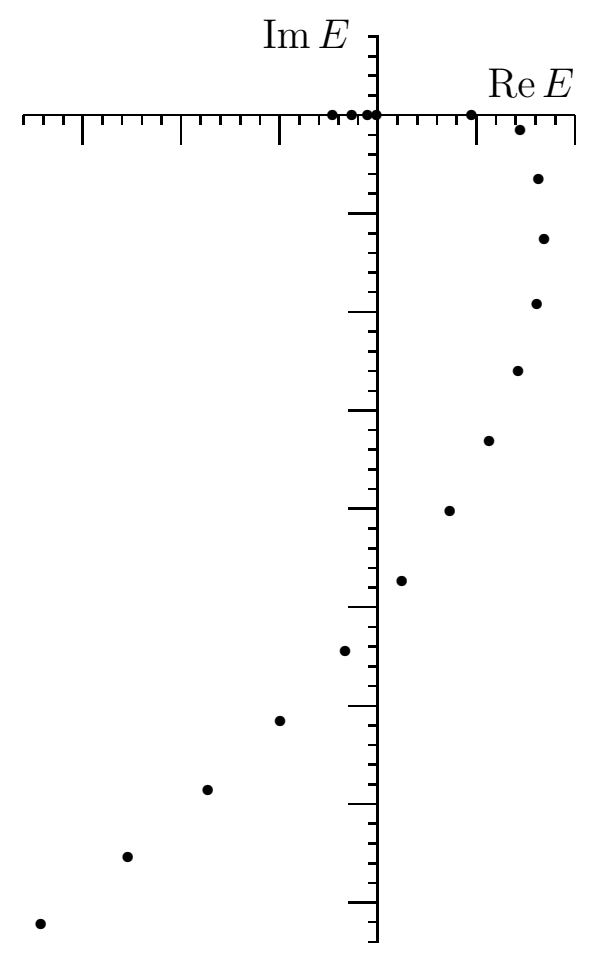

Figure 4: Spectral points generated by the potential (68) and given in Table 1 . 


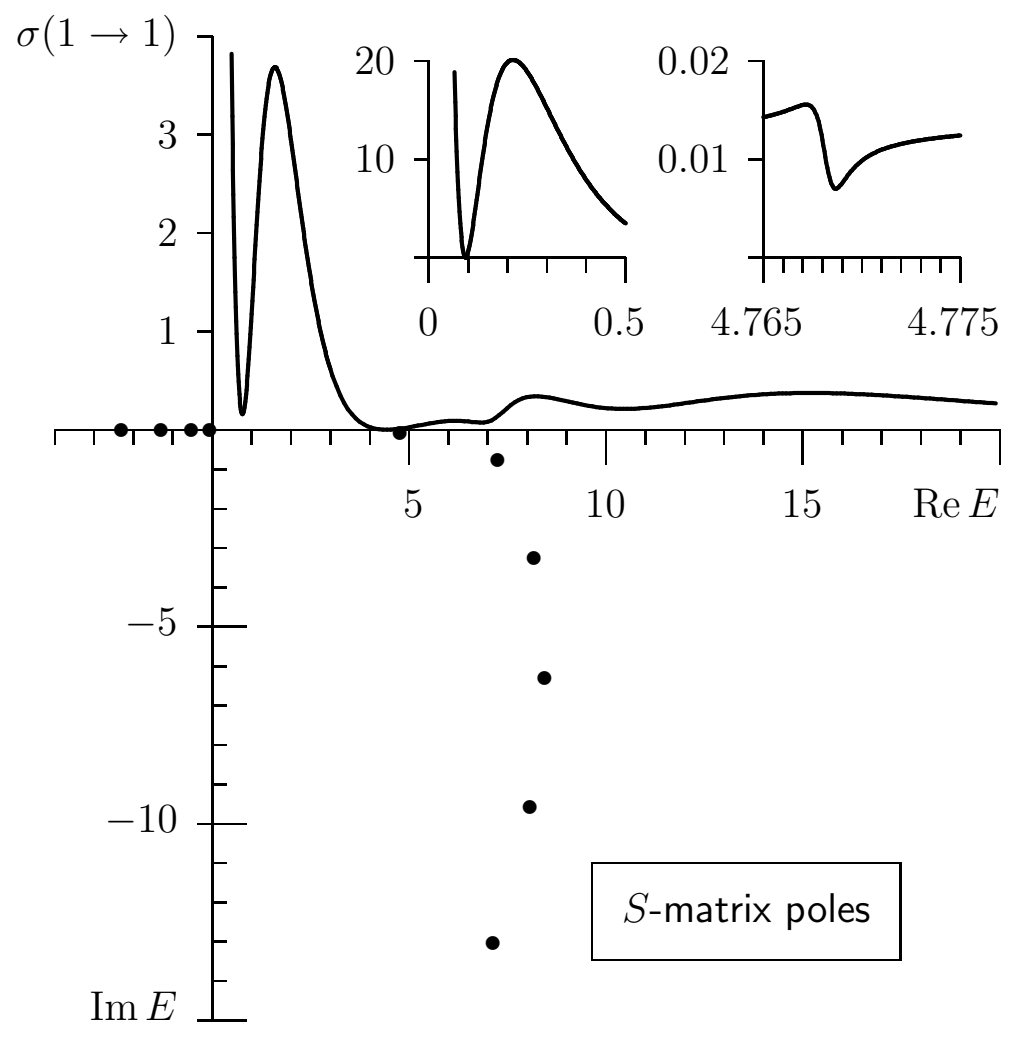

Figure 5: Energy dependence of the elastic scattering cross section in channel 1 for the potential (68). Few of the $S$-matrix poles (see Table 1 and Fig. 44) are shown in the lower part of the Figure. 


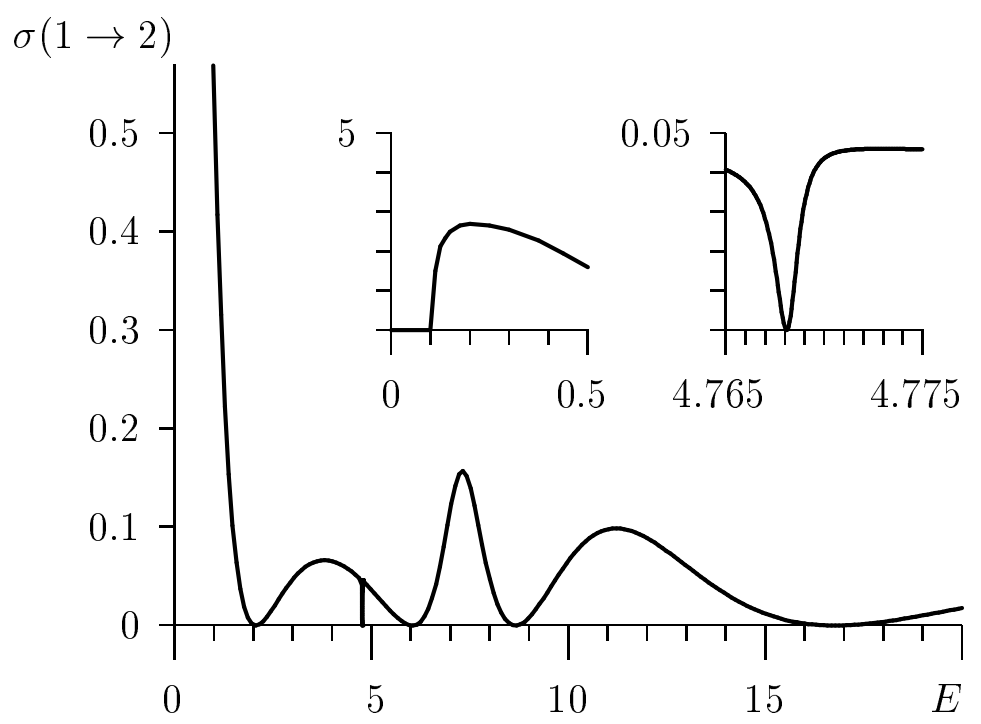

Figure 6: Cross section energy dependence of the inelastic transition $(1 \rightarrow 2)$ for the potential (68).

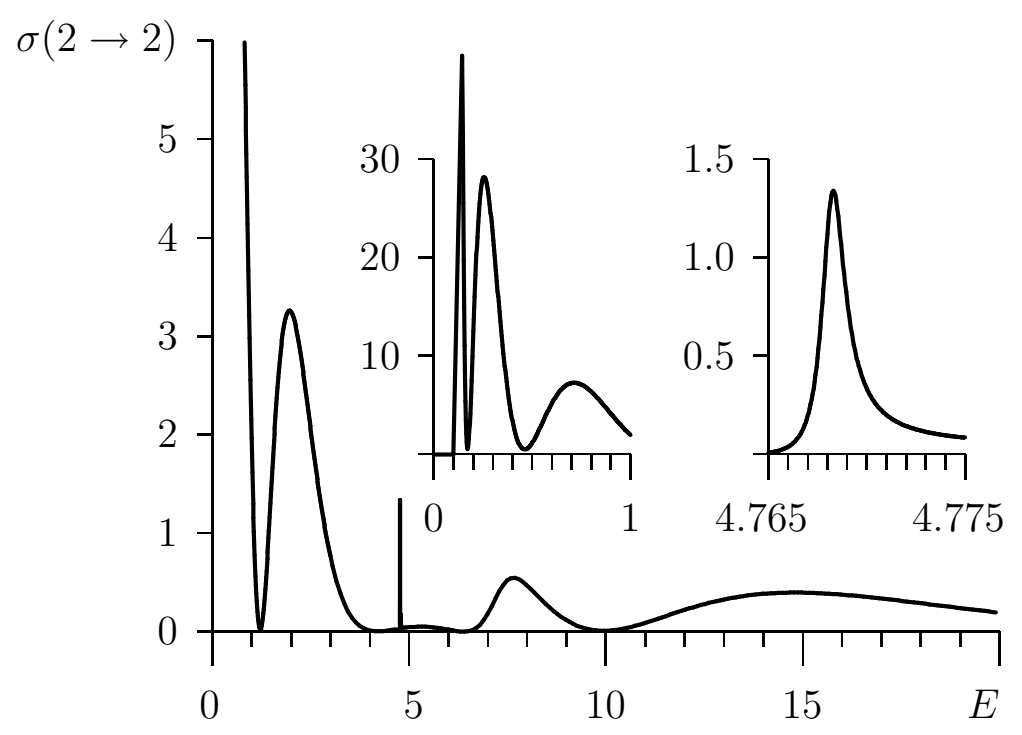

Figure 7: Energy dependence of the elastic scattering cross section in channel 2 for the potential (68). 


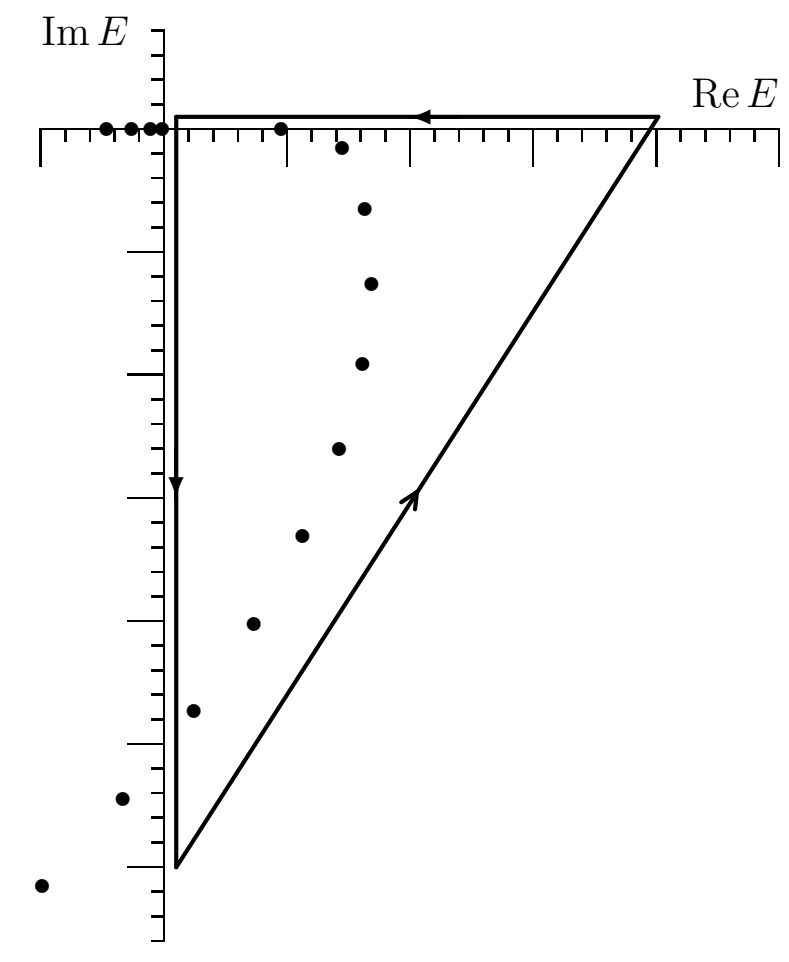

Figure 8: The integration contour for Eq. (70). The vertices of the triangle are $(0.5,0.5)$, $(0.5,-30)$, and $(20.1,0.5)$. 


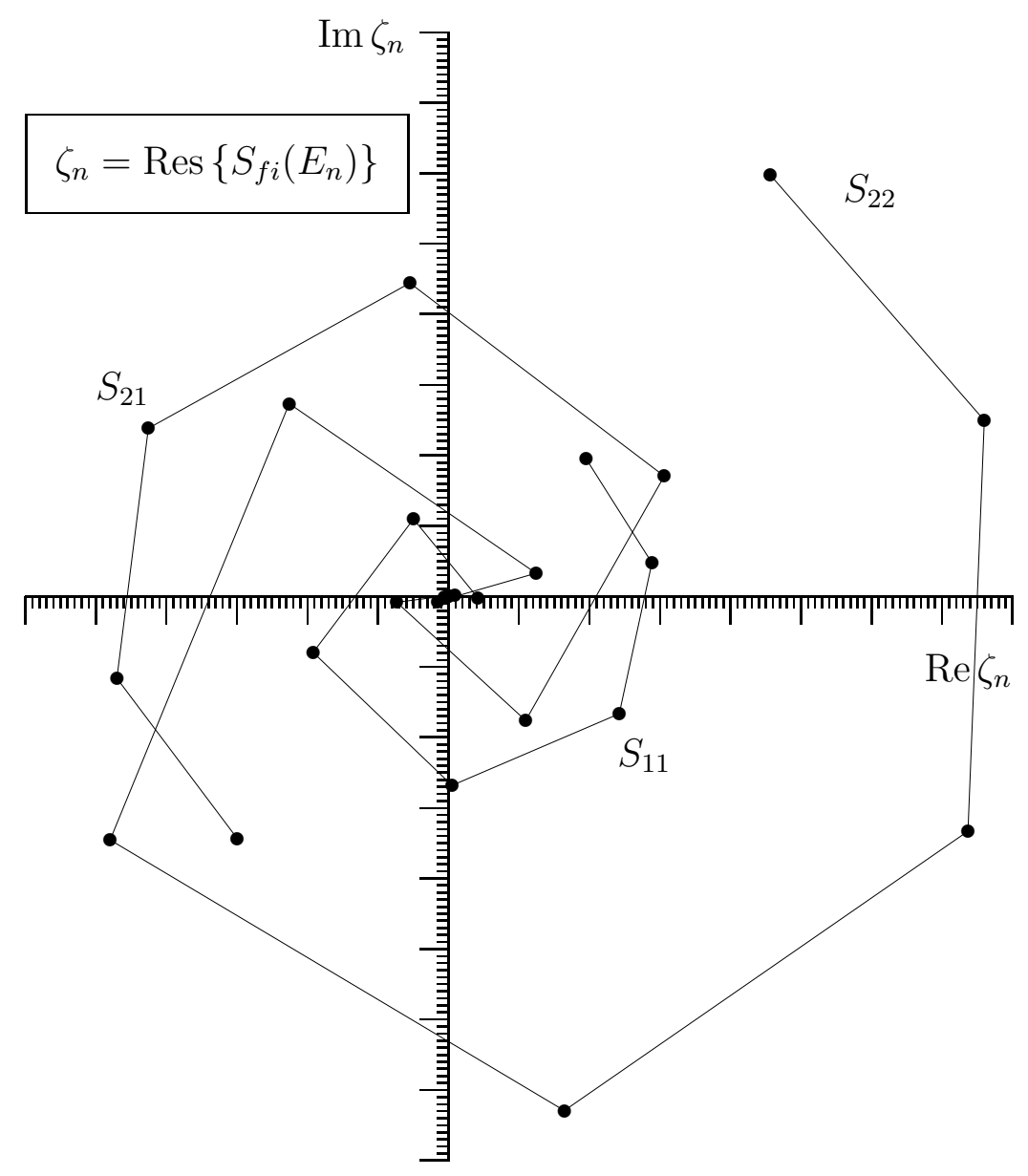

Figure 9: Residues of the $S$-matrix elements at the 9 resonance poles enclosed in the contour shown in Fig. 8. The coordinates of the points are given in Table 2. 


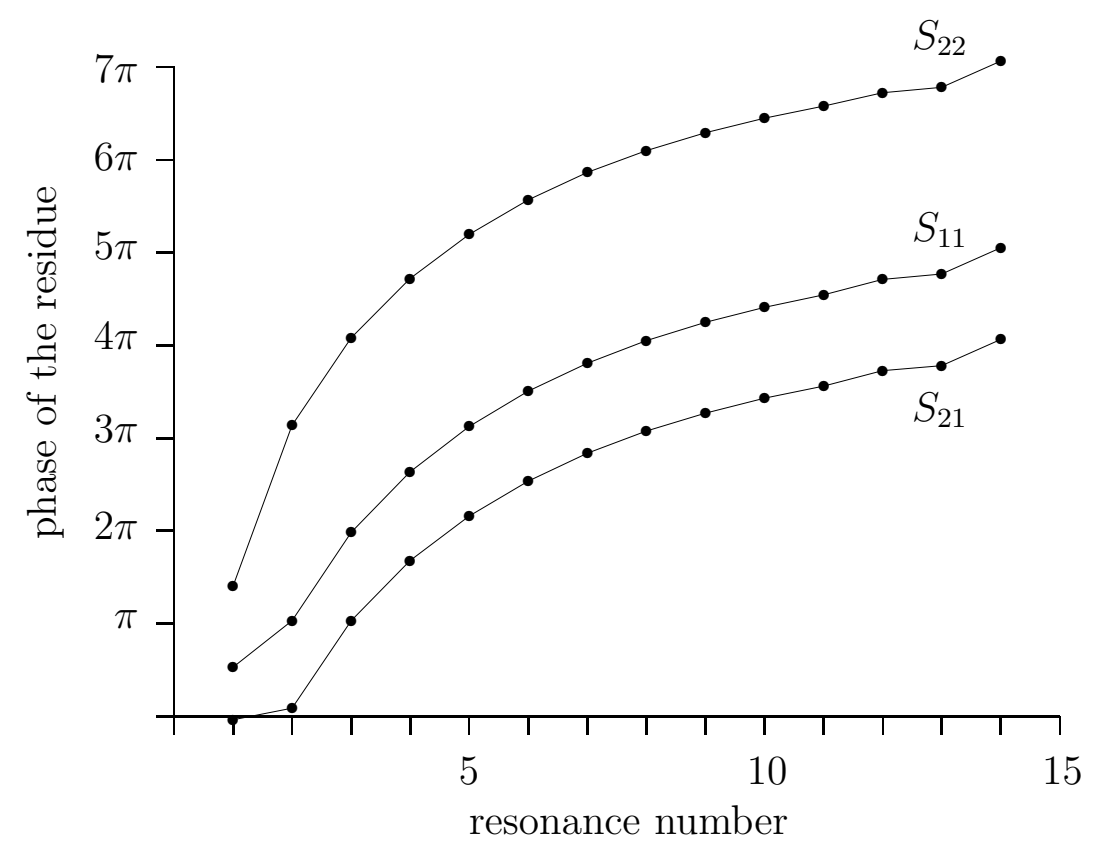

Figure 10: Phases of the residues of the $S$-matrix elements at the first 14 resonance poles given in Table 1 . 


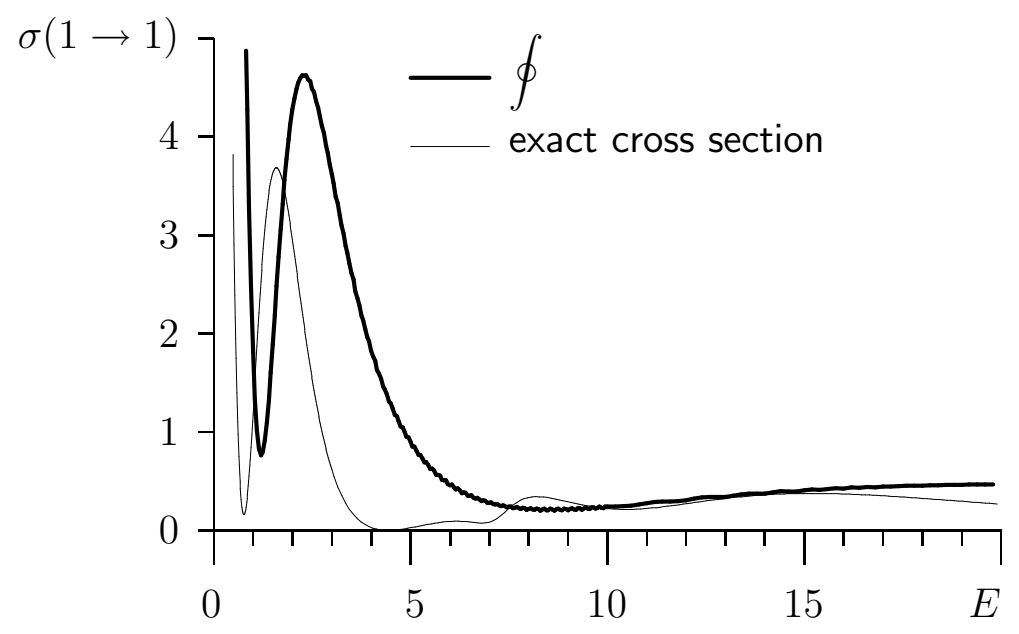

Figure 11: Comparison of the exact cross section for the elastic scattering in the channel 1 (thin curve) with the corresponding cross section obtained from the expansion (71) where all the pole terms are omitted (thick curve).

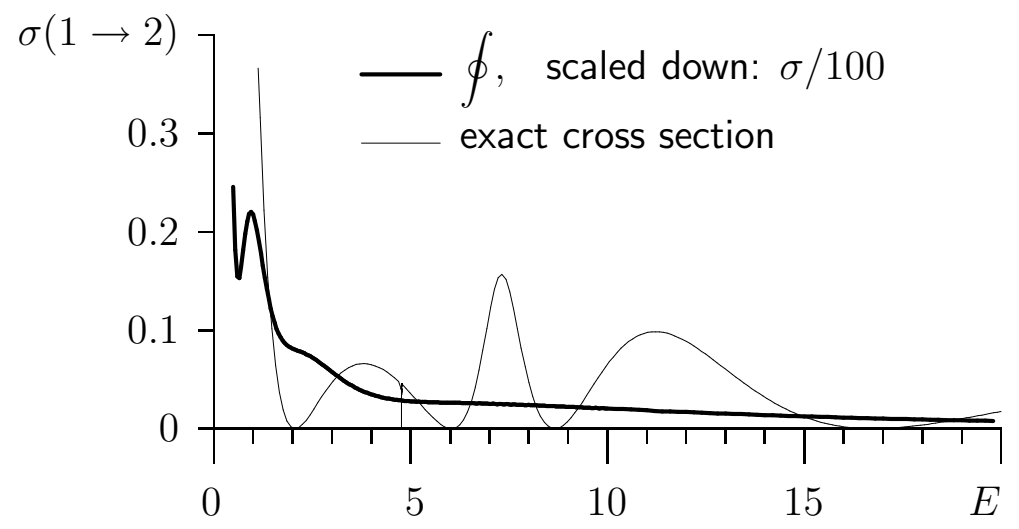

Figure 12: Comparison of the exact cross section for the inelastic transition $(1 \rightarrow 2)$ (thin curve) with the corresponding cross section obtained from the expansion (71) where all the pole terms are omitted. In order to fit into the picture the thick curve is scaled down by the factor of $1 / 100$. 


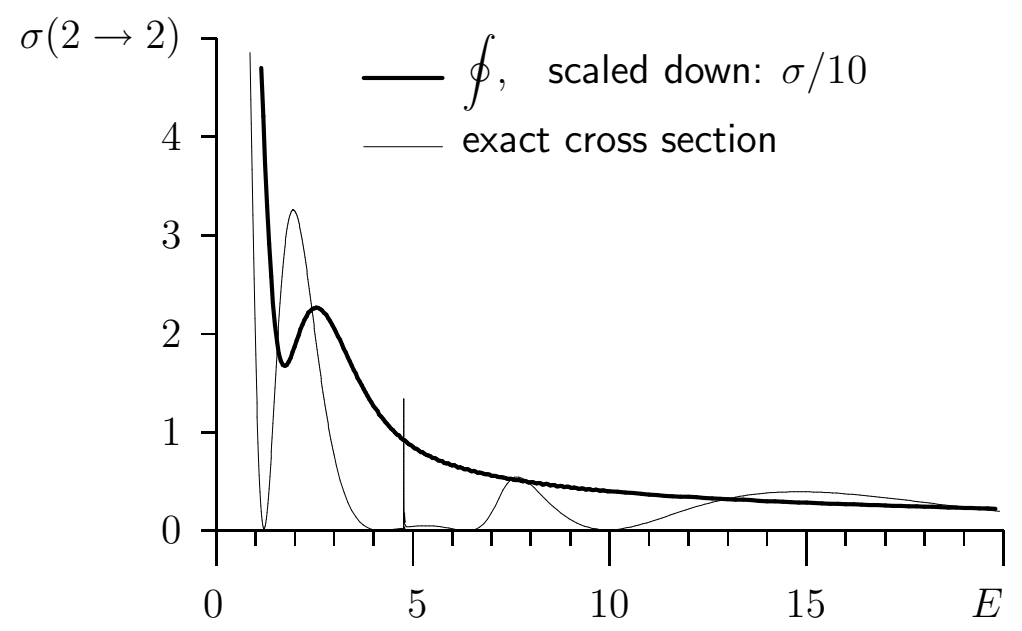

Figure 13: Comparison of the exact cross section for the elastic scattering in the channel 2 (thin curve) with the corresponding cross section obtained from the expansion (71) where all the pole terms are omitted. In order to fit into the picture the thick curve is scaled down by the factor of $1 / 10$. 


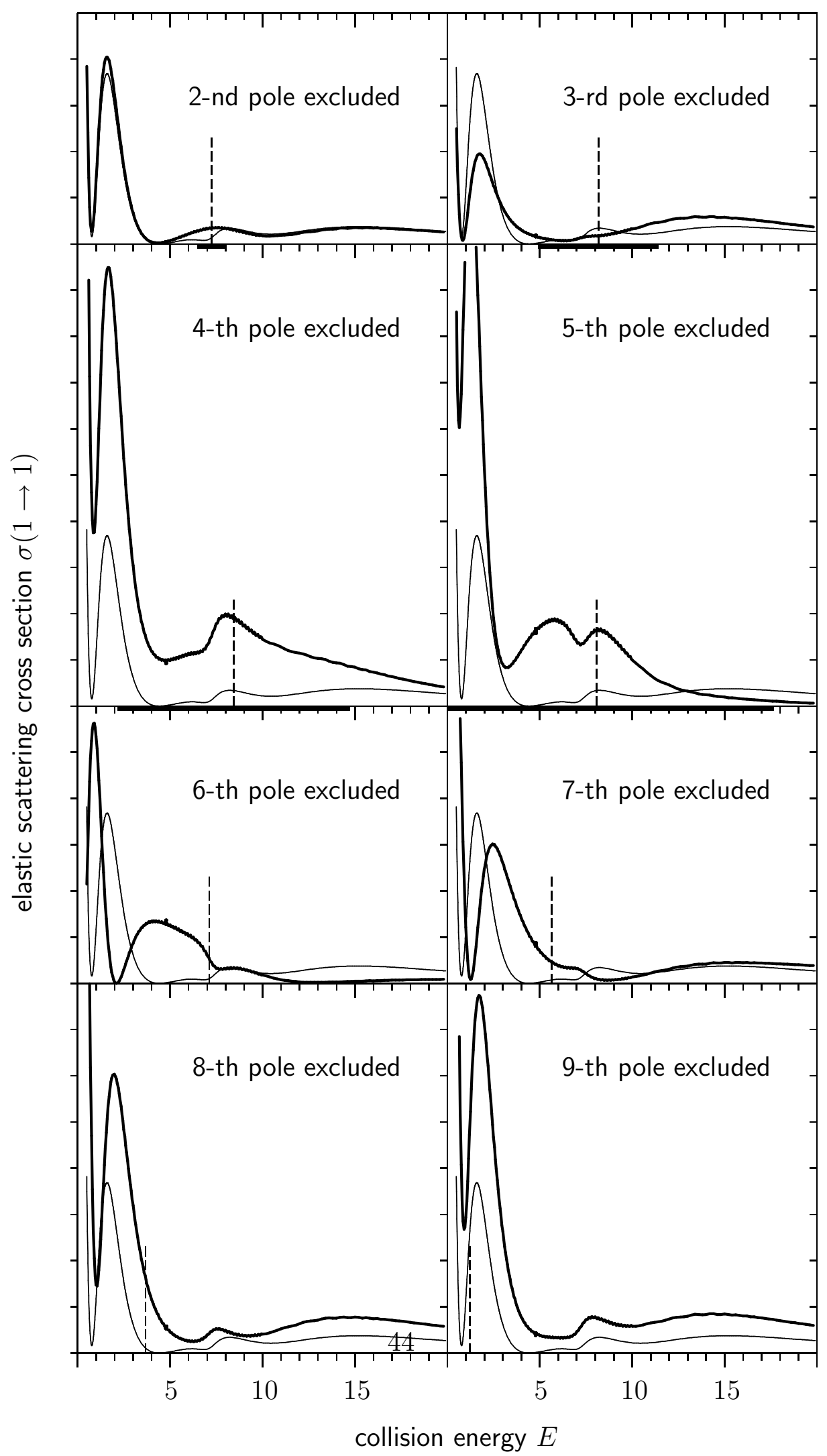

Figure 14: Exact cross section for the elastic scattering in channel 1 (thin curve) and the corresponding cross section obtained from the expansion (71) where one of the nine pole 


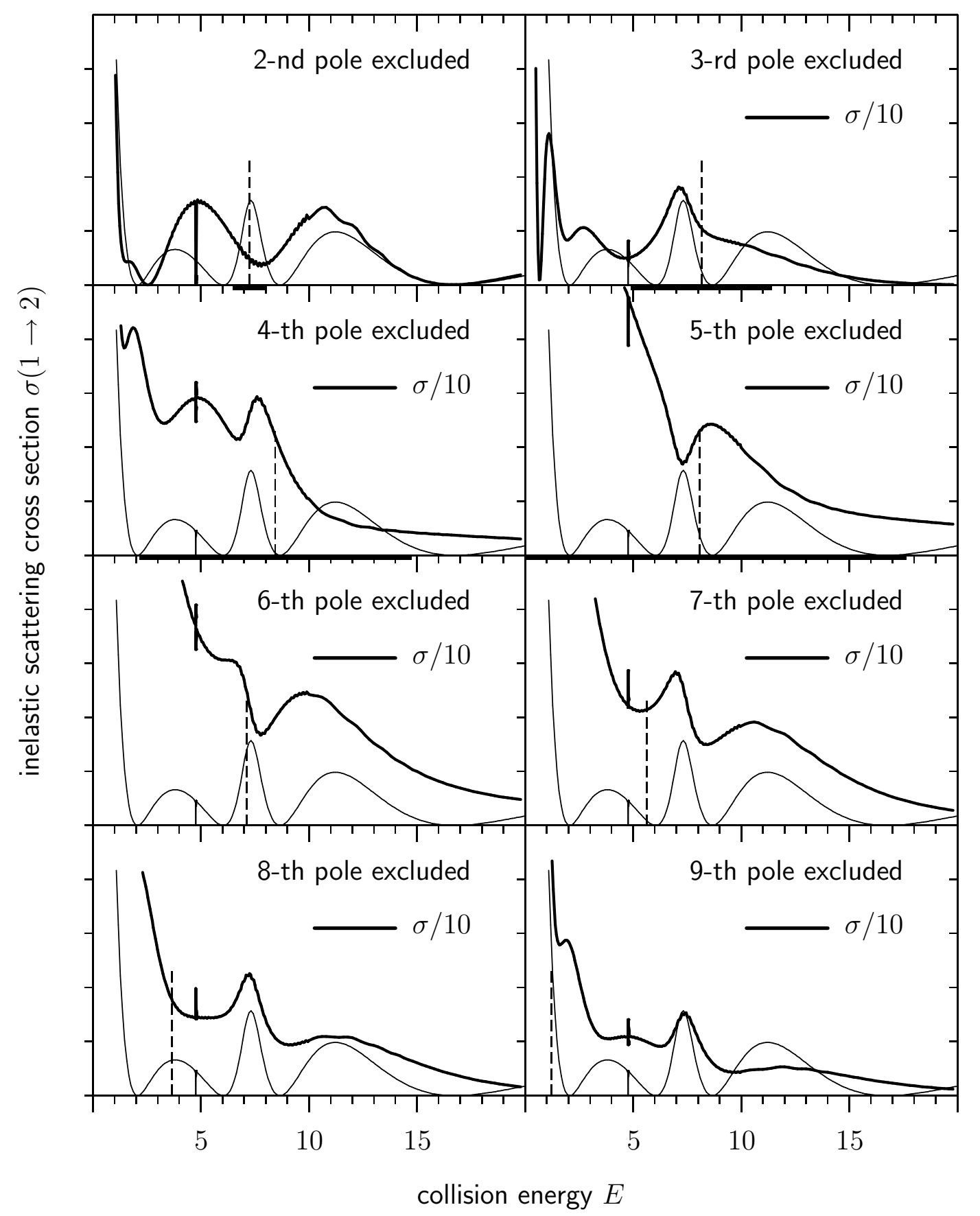

Figure 15: Exact cross section for the inelastic transition $1 \rightarrow 2$ (thin curve) and the corresponding cross section obtained from the expansion (71) where one of the nine pole terms is omitted (thick curve). As indicated on the graphs, some of the thick curves are scaled down by the factor of 0.1 in order to fit into the picture. Vertical dashed lines indicate the positions $E_{\mathrm{r}}$ of the excluded resonances and thick horizontal bars on the energy axis cover the corresponding intervals $E_{\mathrm{r}} \pm \Gamma / 2$. For the last four resonances, such intervals exceed the energy segment shown on the figure. 


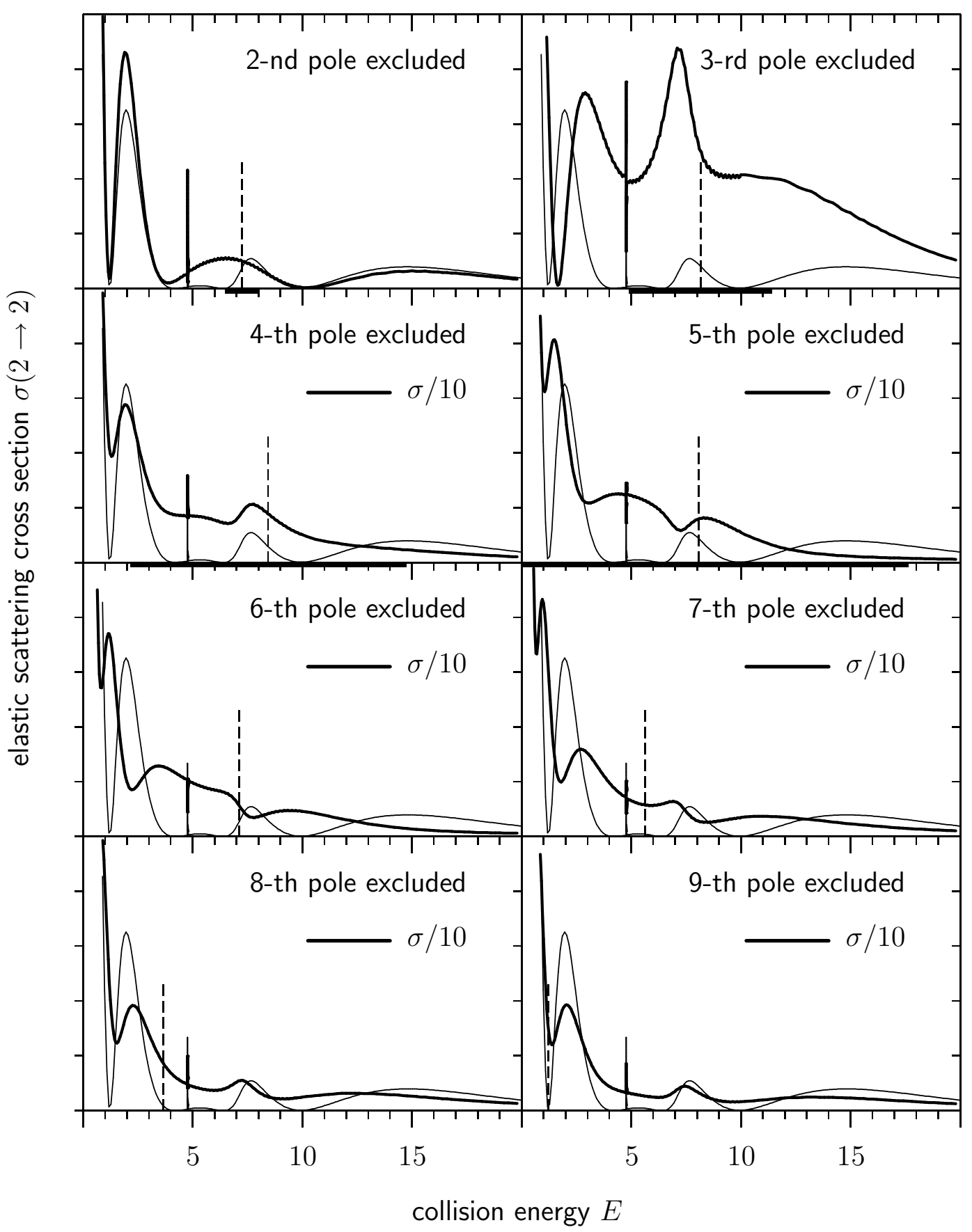

Figure 16: Exact cross section for the elastic scattering in channel 2 (thin curve) and the corresponding cross section obtained from the expansion (71) where one of the nine pole terms is omitted (thick curve). As indicatediøn the graphs, some of the thick curves are scaled down by the factor of 0.1 in order to fit into the picture. Vertical dashed lines indicate the positions $E_{\mathrm{r}}$ of the excluded resonances and thick horizontal bars on the energy axis cover the corresponding intervals $E_{\mathrm{r}} \pm \Gamma / 2$. For the last four resonances, such intervals exceed the energy segment shown on the figure. 


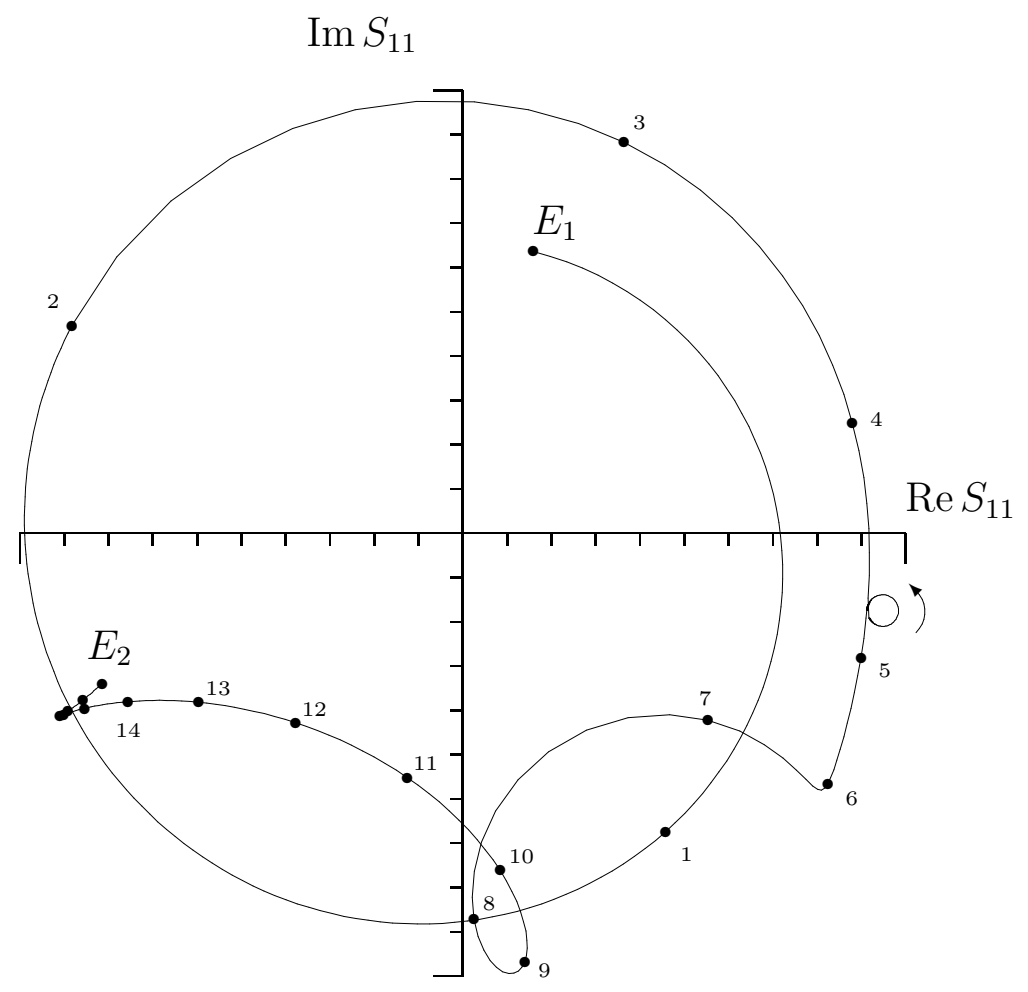

Figure 17: Argand plot of $S_{11}(E)$ in the energy interval from $E_{1}=0.5$ to $E_{2}=20$. The dots on the curve indicate the points corresponding to $E=1,2,3, \ldots$ 


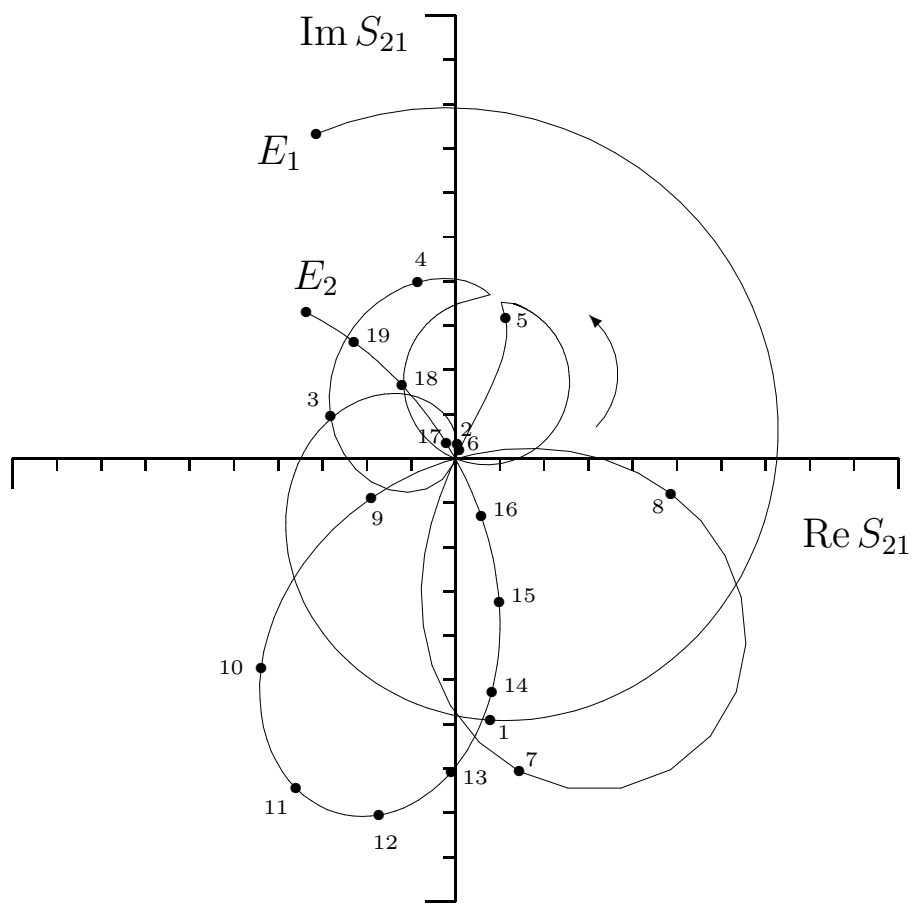

Figure 18: Argand plot of $S_{21}(E)$ in the energy interval from $E_{1}=0.5$ to $E_{2}=20$. The dots on the curve indicate the points corresponding to $E=1,2,3, \ldots$. 


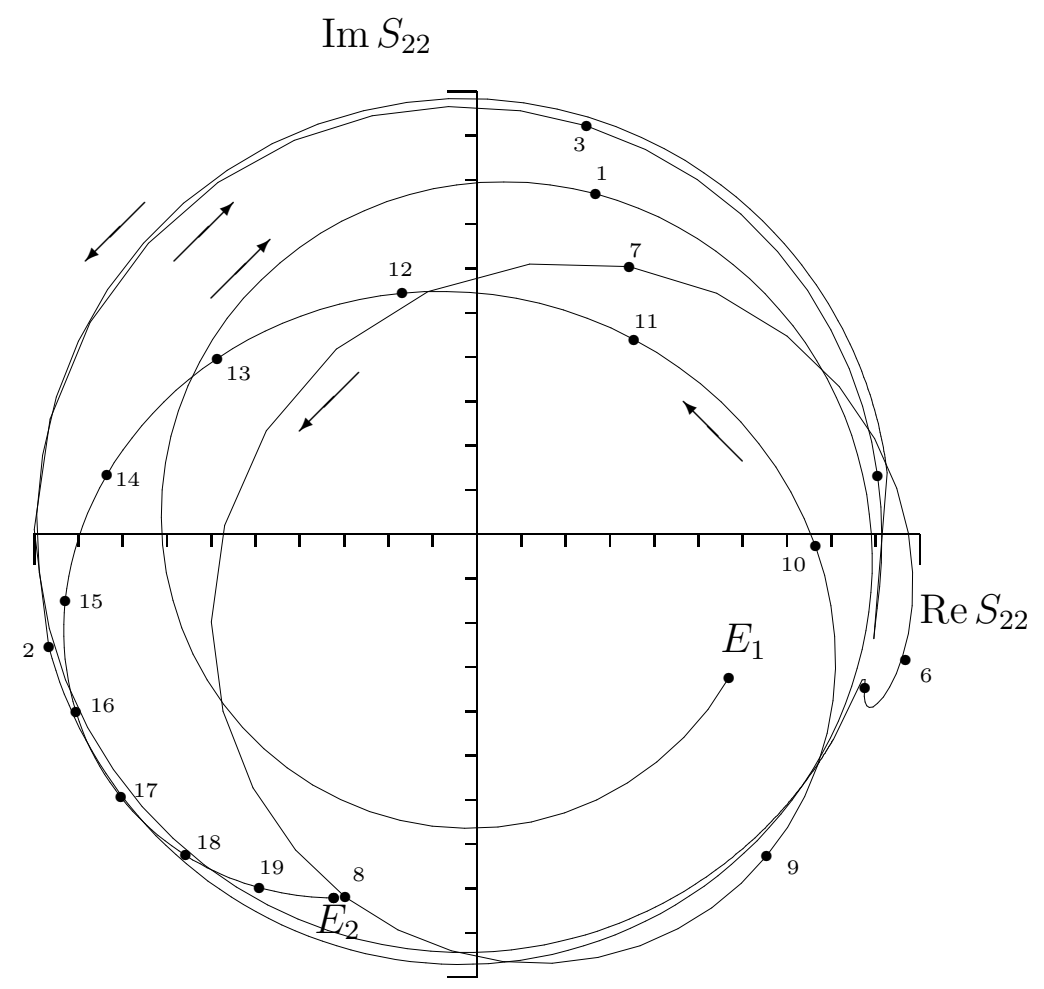

Figure 19: Argand plot of $S_{22}(E)$ in the energy interval from $E_{1}=0.5$ to $E_{2}=20$. The dots on the curve indicate the points corresponding to $E=1,2,3, \ldots$ 


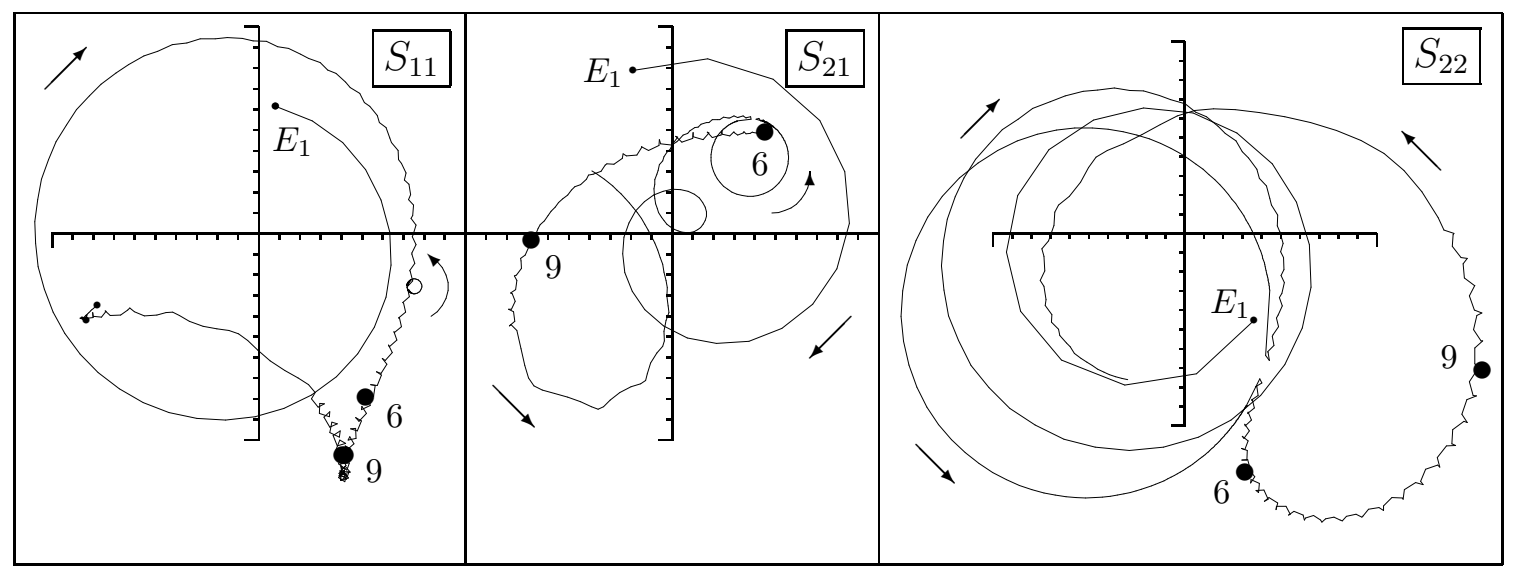

Figure 20: Argand plot of the $S$-matrix with the second pole excluded. The dots mark the points corresponding to $E=6$ and $E=9$.

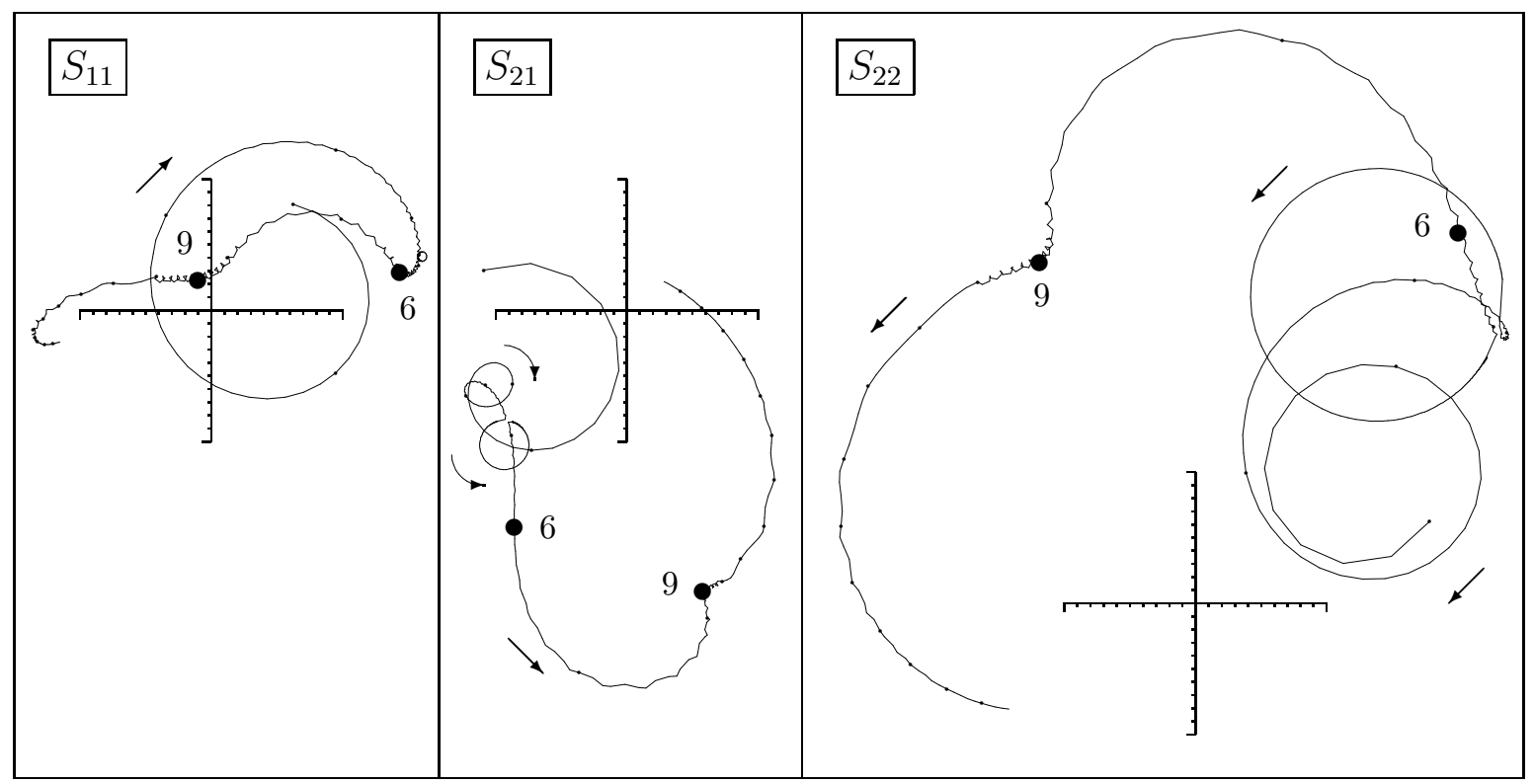

Figure 21: Argand plot of the $S$-matrix with the third pole excluded. The dots mark the points corresponding to $E=6$ and $E=9$. 


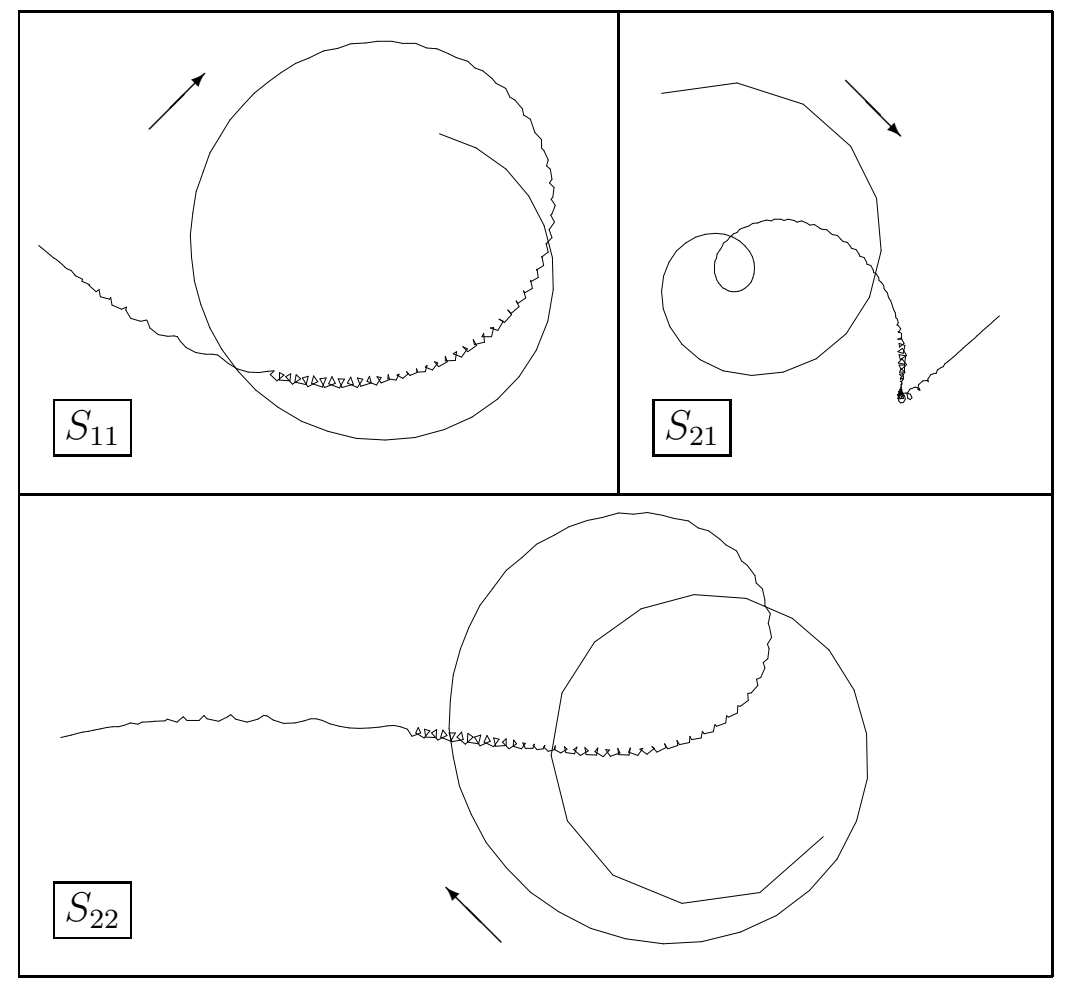

Figure 22: Argand plot of the $S$-matrix with all nine resonance poles excluded. 